\title{
Constraining the abundance of primordial black holes with gravitational lensing of gravitational waves at LIGO frequencies
}

\author{
Jose M. Diego ${ }^{*}$ \\ Instituto de Física de Cantabria (CSIC-UC) Edificio Juan Jordá, \\ Avenida Los Castros s/n, 39005 Santander, Spain
}

(Received 22 November 2019; accepted 18 May 2020; published 15 June 2020)

\begin{abstract}
Gravitational waves from binary black holes that are gravitationally lensed can be distorted by small microlenses along the line of sight. Microlenses with masses of a few tens of solar masses, and that are close to a critical curve in the lens plane, can introduce a time delay of a few milliseconds. Such a time delay would result in distinctive interference patterns in the gravitational wave that can be measured with current experiments such as LIGO/Virgo. We consider the particular case of primordial black holes with masses between 5 and 50 solar masses acting as microlenses. We study the effect of a population of primordial black holes constituting a fraction of the dark matter, and contained in a macrolens (galaxy or cluster), over gravitational waves that are being lensed by the combined effect of the macrolens plus microlenses. We find that, at the typical magnifications expected for observed gravitational wave events, the fraction of dark matter in the form of compact microlenses, such as primordial black holes, can be constrained to the percent level. Similarly, if a small percentage of the dark matter is in the form of microlenses with a few tens of solar masses, at sufficiently large magnification factors, all gravitational waves will show interference effects. These effects could have an impact on the inferred parameters. The effect is more important for macroimages with negative parity, which usually arrive after the macroimages with positive parity.
\end{abstract}

DOI: 10.1103/PhysRevD.101.123512

\section{INTRODUCTION}

Gravitational waves (GWs hereafter) open new opportunities to study the Universe. GW detectors have very wide beams and are sensitive to most of the sky. The sensitivity as a function of the sky position is modulated by the geometric factor, that depends on the relative orientation of the GW detector with the line of sight of the event. For one detector with a maximum response of 1 at the zenith/nadir, the fraction of the sky with a response above 0.5 is $64 \%$. This fraction increases to $\approx 90 \%$ for beam responses greater than $30 \%$ of the maximum response. That is, at any given time, a GW detector is seeing $90 \%$ of the sky with a sensitivity greater than $30 \%$ its maximum sensitivity. Rare events can then be observed, provided their amplitude is large enough. Strong gravitational lensing of distant background objects can be considered as a rare event, since the probability that a distant source $(z>1)$ is strongly lensed is less than $0.1 \%$. Observations of transients (like supernovae) that are strongly lensed are extremely rare, since detecting transients has traditionally required one to be observing that particular region of the sky at the time of the transient, and only a small fraction of these transients are expected to be strongly lensed. In the case of GWs, one does not need to

jdiego@ifca.unican.es be pointing a telescope to a particular region in the sky. Then, if a distant GW is being strongly lensed, it does not fall in the relatively small blind-spot regions in the sky of the detector, and is magnified above the detection threshold of the detector, it will be observed independently of its sky position, since the GW detector has a wide beam extending over the entire sky (but modulated by the geometric factor). As the number of available detectors grows, the overlapping beams will result in a more uniform sensitivity across the sky, removing blind spots and making detectability even more likely.

If the rate of GWs is sufficiently high at $z>1$, the number of GWs per redshift interval at large redshifts (that scales to first order as $z^{3}$ ) may be large enough to compensate for the small probability of lensing. Then, some of the events that could not be observed at $z>1$, because they are too distant, could be promoted beyond the sensitivity threshold of the detector if they are magnified by some factor. Strong lensing of GWs is different than standard lensing of galaxies in different ways. Owing to the very low frequency of the GWs, and depending on the mass of the lens, diffraction effects may be important. This will be studied in more detail in the sections below. On the other hand, due to the very small size of the volume emitting GWs (basically, the volume containing the compact binary), magnification factors of many thousands are 
possible. This is not true in the case of strongly lensed galaxies, where magnification factors above a few tens are rare. This is due to the fact that the maximum magnification is proportional to $\sqrt{R}^{-1}$, where $R$ is the radius of the object being lensed. Allowing for magnification factors larger than a few hundred has important implications on the detectability of distant objects. GWs that are emitted at a redshift $z \approx 3$, if magnified by a factor of $\approx 300$, can compensate the increase in the luminosity distance and appear as luminous as a similar event at $z \approx 0.3$. On the contrary, the galaxy hosting this GW would have its total flux magnified only by a factor of a few tens at most.

A high rate of events at high redshift may require the existence of an exotic compact object candidate for dark matter: the primordial black holes (or PBHs hereafter). PBHs have been hypothesized as a possible candidate for dark matter [1-6]. Rates of events (with masses $\approx 30 \mathrm{M}_{\odot}$ ) as high as $10^{4}$ events per $\mathrm{Gpc}^{3}$ and year (at $z \approx 1-2$ ) are possible if the fraction of dark matter in the form of a $\mathrm{PBH}$ is of the order of $10 \%$ [7]. If a fraction of the dark matter is composed of PBHs, they will not only have an impact on the rate of merger events, but can also act as microlenses and split an incoming GW into multiple images with a time delay between them. If the mass of the microlens is in a certain range, diffraction effects may take place on the GW $[8,9]$. The GW may interfere with itself if the time delay between the lensed microimages is of the order of the period of the GW. Lensing of GWs by compact astrophysical bodies has been considered in the past, although normally earlier work considers masses above $100 \mathrm{M}_{\odot}$ [10-14]. In Ref. [15], the authors consider lensing by PBHs with masses between $\sim 10$ and $\sim 10^{5} \mathrm{M}_{\odot}$. In that work, the lensing probability is computed assuming a simple model in which all microlenses are isolated; that is, the halo (galaxy, group, or cluster) hosting the microlens is being ignored. As shown by Ref. [16], the role of the macrolens cannot be ignored, since it can modify significantly the time delays and, consequently, the detectability of the microlensing event. The calculation of the optical depth of lensing is also affected by the macrolens. This is particularly important for the most distant detected GWs, most of which will be strongly lensed. At large magnification factors, the probability of microlensing grows as the magnification of the macrolens [17]. As discussed in Ref. [16], at sufficiently large magnifications, all strongly lensed GWs will be affected by microlensing. This happens when the effective optical depth is $\approx 1$.

In this work, we focus on lensed binary black holes (BBHs), although the results presented in this paper are also relevant for other GWs at higher frequencies like binary neutron stars (BNSs) or neutros star black hole. However, since these alternative candidates have a smaller chirp mass, the probabilities of observing these events at higher redshifts is smaller. As demonstrated in earlier work, and also shown in the sections below, the probability of strong lensing is sufficiently high only at redshifts above 0.5. BBHs are luminous enough that, after being magnified by some factor, they can be observed even if they are produced at $z>1$.

Under the hypothesis that dark matter is composed of PBHs with masses around 30 solar masses, GWs that are being strongly lensed by massive halos are useful probes of dark matter. Events that are strongly lensed necessarily must travel through areas with a relatively large surface mass density. As strong lensing events must take place near the critical curves of halos, the surface mass density near critical curves is close to the critical surface mass density for lensing, which typically is in the range of a few thousand solar masses per parsec square. This means that, in projection, a parsec square must contain dozens of PBHs in the intervening macrolens alone (and more when one accounts for additional matter along the line of sight).

We adopt a cosmological model with $\Omega_{m}=0.3$, $\Lambda=0.7$, and $h=0.7$. A few terms will be used throughout the paper. We refer to the massive halos (galaxies, groups, and clusters) as macrolenses. The stars, remnants, and PBHs in these macrolenses that perturb the macrolens on the small scale are referred to as microlenses. The images produced by the macrolens are referred to as macroimages. In the presence of microlenses in the lens plane, macroimages of small sources can break up into smaller microimages around the positions of the microlenses. The source frame is the reference frame where the GW is originated, while the observer frame is Earth. The structure of this paper is as follows. In Sec. II, we give a brief introduction to lensing of GWs in the regime of geometric optics. This regime is the appropriate one to address the probability of lensing of a GW by a macrolens, since the Schwarzschild radius of the lens is in this case much larger than the wavelength of the GW (this is the criterion that is normally used to separate between geometric and wave optics). Section III introduces the chirp mass function of GWs. Section IV discusses the probability of lensing by macrolenses. In Sec. V, we describe two models for the evolution of the rate of GWs as a function of time. Section VI estimates the expected number of strongly lensed events for the two models assumed. In Sec. VII, we discuss the effects that microlenses have on GWs, including wave effects that, at LIGO frequencies, become relevant for microlenses with masses below a few hundred $\mathrm{M}_{\odot}$. In Sec. VIII, we describe the simulation used to test the sensitivity of microlensing of GWs to the abundance of PBHs. Finally, in Sec. IX, we present the main results of this work and discuss its implications.

\section{LENSING OF GRAVITATIONAL WAVES}

Lensing of GWs has been studied in detail in earlier work [10-12] and more recently in Refs. [15,18-24]. In general, wave optics is the appropriate regime for studying the lensing of GWs when the Schwarzschild radius of the 
lens is comparable to the wavelength of the $\mathrm{GW}[9,11,25]$. For the frequencies probed by experiments such as LIGO/ Virgo, wave optics is relevant when the lens has a mass smaller than a few thousand solar masses. For lenses with masses above $10^{4}$ solar masses, one can rely on geometric optics. We discuss the regime of wave optics in Sec. VII. This section presents only a brief discussion of lensing of GWs in the regime of geometric optics.

The most basic quantity that can be measured from a GW is the observed (redshifted) chirp mass

$$
\mathcal{M}=(1+z) \mathcal{M}_{\text {chirp }}=(1+z) \frac{\left(m_{1} m_{2}\right)^{3 / 5}}{\left(m_{1}+m_{2}\right)^{1 / 5}},
$$

where $m_{1}$ and $m_{2}$ are masses of the two objects before coalescence (in the source frame) and $\mathcal{M}_{\text {chirp }}$ is the chirp mass in the source frame.

Only GWs that are above the detection threshold of the detector can be observed. This threshold is determined by the signal-to-noise ratio $\rho$ of the GW. To first order, the value of $\rho$ depends on the luminosity distance of the $\mathrm{GW}$, $D_{l}(z)$, and the observed (i.e., detector-frame) chirp mass $\mathcal{M}$. More specifically, $\rho$ is obtained after integrating the square of the Fourier transform of the observed signal over a frequency range and weighted by the inverse of the power spectral density of the detector (see, for instance, $[26,27]$ ):

$$
\rho=\sqrt{\mu} \frac{\mathcal{M}^{5 / 6}}{D_{l}(z)} \sqrt{\mathcal{F}(\Theta, s, p, \theta) \zeta\left(f_{\max }\right)} .
$$

The term $\mathcal{F}(\Theta, s, p, \theta)$ accounts for the geometric configuration, including the angular position in the sky, orientation of the detector, spin, polarization, and orbital inclination of the binary. The term $\zeta$ encapsulates the detector response through the noise spectral density and the maximum frequency of the $\mathrm{GW}, f_{\max }$. This maximum frequency (twice the frequency of the last orbit) is determined by the innermost stable circular orbit (ISCO). The ISCO frequency scales as the inverse of the total mass of the binary system [27-29]. Larger chirp masses correspond to smaller $f_{\max }$ and, consequently, to smaller $\zeta$, partially compensating the dependency of $\rho$ with $\mathcal{M}^{5 / 6}$. More specifically, the term $\zeta$ scales as $\int_{f_{\min }}^{f_{\max }} d f f^{-7 / 3} / \mathcal{S}(f)$ [27], where $S(f)$ is the power spectral density of the detector. For the range of frequencies and sensitivity in LIGO, one can use the published $\mathcal{S}(f)$ for the observed LIGO events to estimate $\zeta$. For the typical BBH events detected by LIGO, $\zeta$ has a mild dependency with $f_{\max }$. In particular, for the published $S(f)$ curves in LIGO, $\zeta$ scales roughly as $f_{\max }^{0.1}$ (or as $\mathcal{M}^{-0.1}$ ) for $f_{\max }$ between 300 and $700 \mathrm{~Hz}$, which is the typical range of the observed BBHs in LIGO. This modest dependency with $f_{\max }$ is due to the fact that most of the power of the signal is contained in the lower frequencies, so, above $200 \mathrm{~Hz}$, the contribution to $\rho$ is relatively small. The focus of this paper is on the often neglected first term in Eq. (2). This is the square root of the magnification, $\mu$, which is usually assumed to be $\mu=1$, and hence ignored [30]. If a GW is being amplified by strong lensing, by ignoring $\mu$, the inferred luminosity distance will be smaller than the true distance by a factor of $\sqrt{\mu}$ and the inferred chirp mass (in the rest frame) larger in order to maintain $\mathcal{M}$ constant. This mechanism has been invoked in the past to explain the unusually high chirp masses observed for some of the events detected in LIGO/ Virgo [19].

\section{CHIRP MASS DISTRIBUTION OF THE BACKGROUND POPULATION}

A fundamental ingredient in this work is the distribution of chirp masses. Little is known about this distribution. The observed events by LIGO/Virgo are consistent with standard power-law models $\left(M^{-2.3}\right)$ but also with a bimodal function having a peak at high masses $\left(\right.$ at $\approx 40 \mathrm{M}_{\odot}$ ) [31]. In Ref. [19], the authors make the interesting suggestion that the peak in the bimodal mass function could be the consequence of lensing of the GWs. Since a lensed GW can be misinterpreted (if lensing is ignored) as a closer $\mathrm{GW}$ with a larger chirp mass, lensed GWs could naturally produce observed shallow or even bimodal mass functions. In this work, we assume a simple model for the chirp mass function. We start from a standard stellar model where heavy stars follow the standard power law $M^{-2.3}$, appropriate for masses above $1 \mathrm{M}_{\odot}$ [32], and assume that the remnants (BHs) left by these massive stars have a mass that is proportional to the parent star. Individual masses $\left[m_{1}\right.$ and $m_{2}$ in Eq. (1)] are drawn from this distribution (with $m_{1}$ and $m_{2}$ in the range $5 \mathrm{M}_{\odot}<m_{1}, m_{2}<50 \mathrm{M}_{\odot}$ ), and the chirp mass is computed from the randomly generated $m_{1}$ and $m_{2}$. This approach ignores possible correlations between $m_{1}$ and $m_{2}$. The lack of correlation between $m_{1}$ and $m_{2}$ is expected in the capture scenario where two BHs that form far away from each other form a binary pair, as opposed to common envelope formation scenarios where correlations between $m_{1}$ and $m_{2}$ are possible. Another possibility for correlated masses can take place in the center of globular clusters, where mass segregation may result in heavier BHs sinking toward the center of the cluster and forming binaries with correlated masses [33]. The resulting distribution is shown in Fig. 1 as a solid line. The dashed line is the model we use in this work, which is built from a lognormal function $f(\mathcal{M}) \propto \mathcal{M}^{-1} \exp \left((\ln (\mathcal{M})-\eta)^{2} /\left(2 \sigma^{2}\right)\right)$, where $(\eta=1.68, \sigma=0.12)$ for masses below $5.5 \mathrm{M}_{\odot}$, $(\eta=1.7, \sigma=0.3)$ for masses between 5.5 and $8 \mathrm{M}_{\odot}$, and $(\eta=1.68, \sigma=0.12)$ for masses above $8 \mathrm{M}_{\odot}$. Since $\mathcal{M}$ is dominated by the lightest component (between $m_{1}$ and $m_{2}$ ), the resulting distribution peaks at low chirp masses and falls faster with mass than the original mass function. The minimum chirp mass is $4.35 \mathrm{M}_{\odot}$ (when $\left.m_{1}=m_{2}=5 \mathrm{M}_{\odot}\right)$. The resulting mass function resembles the low-mass peak of model C considered in Ref. [31], 


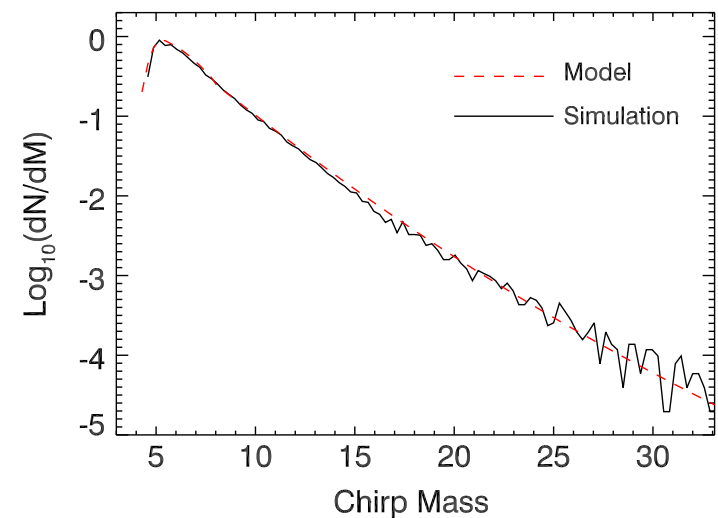

FIG. 1. Mass function for the chirp mass. The solid line shows the result of a simulation where the chirp mass is computed from individual masses following the standard power-law model with exponent -2.3 . The dashed line is a (log-normal) model that reproduces well the simulation as described in the text.

although with a lower mean mass (see Fig. 1 in that reference). It is important to note that, as demonstrated below (and originally proposed by Ref. [19]), the highmass peak of model $\mathrm{C}$ in Ref. [31] can be naturally produced by lensing, provided the rate of events at high redshift is sufficiently high. Note also that model $\mathrm{C}$ considered in Ref. [31] allows for some correlation between $m_{1}$ and $m_{2}$, while we ignore this correlation. However, in the model proposed by Ref. [19], lensed events would appear redshifted. In this case, both the intrinsic (i.e., source frame) $m_{1}$ and $m_{2}$ would be uncorrelated, but the observed $m_{1}$ and $m_{2}$ would be affected by the same factor $(1+z)$. For $z>1$, the observed $m_{1}$ and $m_{2}$ would appear to have a clear correlation due to the common, and relatively large, $(1+z)$ factor. This is explored in more detail in Ref. [34], where the authors explain the observed correlation between $m_{1}$ and $m_{2}$ in terms of lensing.

\section{PROBABILITY OF OBSERVING A LENSED GW}

The probability of lensing is referred to as the optical depth of lensing, $\tau$, and in its most basic form depends on the magnification $\mu$ and the redshift of the source, $z_{s}$, i.e., $\tau\left(\mu, z_{s}\right)$. Different authors have estimated $\tau\left(\mu, z_{s}\right)$ adopting different assumptions. Most estimates of $\tau$ are based on analytic models which have the advantage of flexibility, speed, and achieving high spatial resolution (needed to resolve small areas with larger magnifications). On the negative side, analytic calculations do not account for corrections such as projection effects or substructure that can increase the optical depth. N-body simulations can account for both projection effects and substructure but at the expense of resolution. In this work, we follow Ref. [35] and use an analytic model based on the mass function of Ref. [36] and an elliptical halo model to compute $\tau\left(\mu, z_{s}\right)$. We improve on the work of Ref. [35] and modify the elliptical profile by adding a steeper component in the central region. This component accounts for the baryonic contribution that is important in smaller halos and was neglected in Ref. [35]. We also extend the mass range down to $10^{11} \mathrm{M}_{\odot}$ from the minimum mass of $10^{12} \mathrm{M}_{\odot}$ considered in Ref. [35]. The baryonic contribution makes smaller halos more relevant, and, even though their contribution to $\tau$ is still subdominant, they cannot be ignored. An additional improvement involves computing the optical depth in the image plane rather than in the source plane. In Ref. [35], the author used the optical depth computed in the source plane and later corrected for the multiplicity of lensed images. The optical depth computed in the source plane accounts for the total magnification, and it is the best choice when estimating rates of unresolved images. Since GWs can be resolved in time (two counterimages from the same event will arrive separated by a time interval that can range between a few hours to a few days), the optical depth computed in the source plane has the advantage that no corrections are needed to account for multiple images. It also has the advantage of achieving higher spatial resolution (since there is no need to map the image plane into the smaller source plane). The improvement in spatial resolution translates into an improvement into the maximum magnification that can be computed before limited resolution affects the computation. Our calculation can reach magnification factors of 100 before being affected by resolution effects (even for the smallest halos). Above magnification 100, one can safely extrapolate the optical depth with the standard $\mu^{-3}$ law as $\tau\left(\mu_{>100}, z_{s}\right)=\tau\left(\mu_{100}, z_{s}\right)(100 / \mu)^{3}$.

A caution note is needed here. The optical depth depends on many variables. One of them is the assumed profile for the lenses. Changes in the profile can easily result in differences of a factor of 2 in the optical depth. Much of the literature in optical depth of lensing is based on the spherical isothermal sphere profiles, which are known to increase the optical depth of low-mass halos compared with the Navarro-Frenk-White halos used in this work (see, e.g., Figs. 9-11 in Ref. [37], Fig. 2 in Ref. [38], or Fig. 9 in Ref. [39]). For large-mass halos (which are relevant for

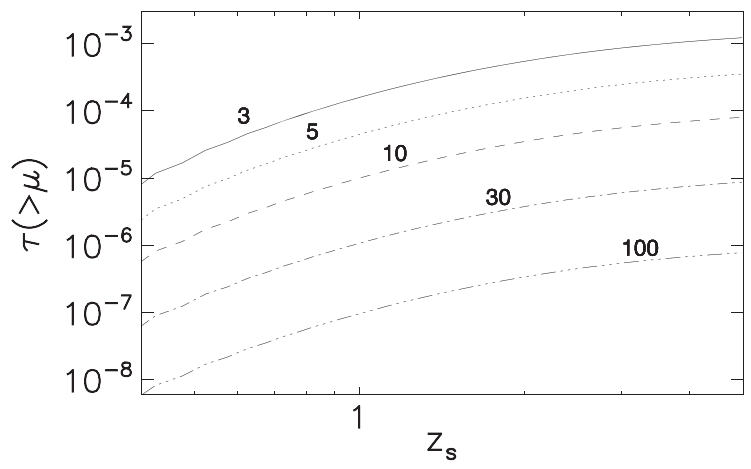

FIG. 2. Optical depth of lensing as a function of the redshift of the background source $\left(z_{s}\right)$ for different values of the magnification (indicated by numbers next to each curve). 
large magnification factors), earlier work based on the velocity dispersion of galaxies fails at capturing the most massive halos (groups and clusters), hence underestimating the optical depth at large magnifications $(\mu>100)$. Finally, the role of baryons is very important, especially for lowmass halos that could be subcritical without baryons but may become supercritical after baryonic collapse. The effect of baryons is not fully accounted for either in analytical models or in N-body simulation calculations. The reader should be wary of these issues and consider the optical depth in this (and other) work(s) as an estimation, subject to an uncertainty of at least a factor of 2. In Fig. 2, we show examples of the optical depth as a function of $z_{s}$ and for different values of the magnification factor. Note how between $\mu=10$ and $\mu=100$ the optical depth has decreased by 2 orders of magnitude, as expected from the scaling $P(\mu) \propto \mu^{-3}$ in strong lensing. This result is similar to the one derived from $\mathrm{N}$-body simulations and ray tracing $[40,41]$.

For magnification values $\mu>1010$, and for redshifts $z_{s}>1$, the probability of lensing is $\approx 10^{-4}$. This number can be confronted with the number of observed strongly lensed quasars (QSOs) (few dozens) out of the total number of known QSOs $\left(>10^{5}\right)$. Between $z=1$ and $z=2$, one expects that one in $\approx 4 \times 10^{5}$ events will be magnified with factors $\mu>30$. Since the volume in this redshift interval is $\approx 450 \mathrm{Gpc}^{3}$, if the rate of GWs above $z=1$ is above 1000 events per $\mathrm{Gpc}^{3}$ and year, one would expect to observe at least one strongly lensed event above $z=1$ per year and with $\mu>30$. The rate of events as a function of redshift is discussed in Sec. V.

\section{RATE EVOLUTION OF BBH}

The rate of GWs as a function of the redshift is unknown and depends on the formation mechanism of the BBH. The simplest models rely on the assumption that the rate of GW events traces the star formation rate. We adopt the model of Ref. [42] as a conservative one [and we denote it as the star formation rate (SFR) case]. This model predicts that the evolution of the rate of GWs is mild in redshift and peaks at $z \approx 2$. We normalize this model at redshift $z=0$ to a rate of a few tens of events per year and $\mathrm{Gpc}^{3}$, consistent with the inferred rate from the LIGO Collaboration ([31], under the assumption of no lensing). This model is shown as a dashed line in Fig. 3. As an alternative case [inspired by Ref. [19] and that we refer to as the Broadhurst-Diego-Smoot (BDS) model], we consider also a model in which lensed GWs occur more frequently at high redshift and has the attractive feature of explaining the apparent observed bimodality of the mass function of $\mathrm{BHs}$, as well as the unexpectedly high number density of very massive events (with chirp masses of $30 \mathrm{M}_{\odot}$ or above). In this model, the rate of events at high redshift (where the lensing probability is highest) is increased by $\approx 2$ orders of magnitude (but still comfortably

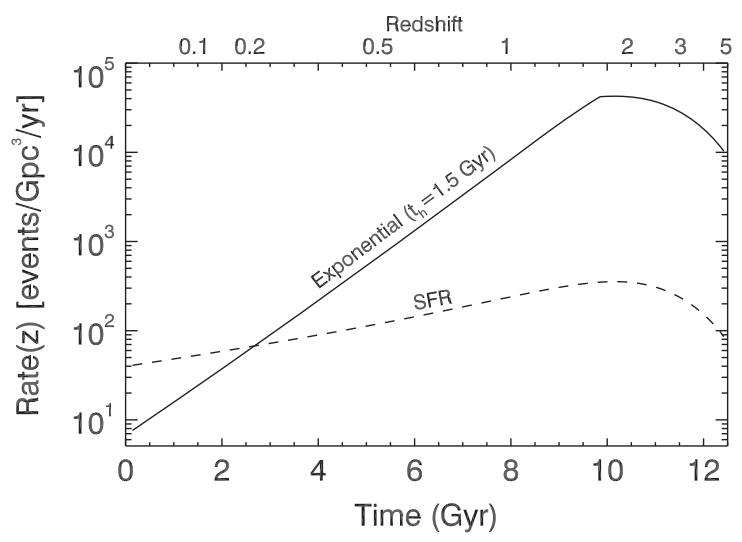

FIG. 3. Rate of events as a function of (lookback) time. The solid line represents a model that grows as the star formation rate at high redshift, but below redshift $z \approx 1.8$ it falls off exponentially with a half-life time of $1 \mathrm{Gyr}$ (in the main text, we refer to this model as the BDS model). The dashed line is a model that traces the star formation rate history. For comparison, the volumetric rate of $\mathrm{SNe}$ (of all types) at $z \approx 2$ is approximately $10^{6}$ per year and $\mathrm{Gpc}^{3}$.

below the rate of $\mathrm{SNe}$ at all redshifts) in order to compensate for the low probability of lensing. To keep the observed rate of events comparable to the ones predicted by the conservative model, the rate at the lowest redshifts must be smaller. We attain this by allowing the rate to decay exponentially between the maximum of the star formation rate at $z \approx 2$ and $z=0$. Using a half-life time of $1.5 \mathrm{Gyr}$ results in a similar predicted number of observed events for both models, so we adopt this value. The fast decline in the rate can be justified by the rapid evolution of $\mathrm{BBHs}$ if these are formed in dense environments like globular clusters (see, e.g., [33]). In this case, the BHs are formed after the SN explosion of the parent star (typically a short-lived massive star), and through mass segregation they sink toward the center of the cluster (since, after the massive stars undergo $\mathrm{SN}$, the most massive objects in the globular cluster are still their remnants). Typical relaxation times are 1 Gyr or less [43-45]. The duration of the phase between the $\mathrm{BBH}$ formation and the final coalescence is more uncertain and depends on mechanisms like dynamical friction and three-body interactions. Most models agree that, once the binary is formed, only a small fraction of the binaries will merge within a Hubble time due to radiation of GWs (see, for instance, [46]). Other mechanisms may need to be invoked to explain a rapid evolution in the merger rate between the peak of star formation and redshift $z=0$.

Also, it is important to consider the fact that massive $\mathrm{BHs}$ are typically produced from massive stars with low metallicity [47]. Below $z \approx 2$, massive stars with low metallicity are expected to be exceedingly rare. Hence, one may expect that BBHs with elevated chirp masses are mostly produced at high redshifts and, if the time of $\mathrm{BBH}$ formation and coalescence is relatively short, a rapid 
evolution between redshift 2 and redshift 0 is expected. Above $z \approx 2$, the rate decays with the redshift in a similar fashion as in the SFR model.

The BDS model is shown as a solid line in Fig. 3. Note that we choose to express the rate as a function of the lookback time in order to accentuate the extended period up to the epoch of maximum star formation rate. Interestingly, the LIGO Collaboration finds some evidence for a rapid evolution in the rate $\left[\mathcal{R} \propto(1+z)^{6.5}[31]\right]$ when GW170729 is included in their analysis. On the contrary, if GW170729 is excluded, the rate is consistent with a nonevolving law. However, when the $90 \%$ confidence regions are accounted for, the rapid evolution model is also consistent with no evolution, reflecting the large degree of uncertainty on the redshift dependency of the merger rate. Note that the BDS model is consistent with model $\mathrm{C}$ in the above reference when the ten BBHs from $\mathrm{O} 1$ and $\mathrm{O} 2$ are included in the analysis (see the redshift evolution model in Fig. 6 in Ref. [31]). On the other hand, it is worth noticing that the relatively high rate of events at high redshift of this model may be in tension with upper limits on the stochastic background of GWs [48]. However, the sensitivity of LIGO to the stochastic background is still $\approx 2$ orders of magnitude above the current estimate of this background [49], so a model such as BDS is still marginally consistent with the lack of detection of this background. Moreover, since GWs at low redshift (with a lower rate in the BDS model at $z<2$ ) contribute more to the stochastic background, a model such as the BDS model is expected to produce a background less than 2 orders of magnitude above the model used in Ref. [49].

\section{EXPECTED NUMBER OF STRONGLY LENSED EVENTS}

With the ingredients presented in the previous sections, we can now compute the expected number of strongly lensed events. This number is basically given by the integral

$$
\frac{d N}{d V d z}=\mathcal{R}(z) \int_{M_{a}}^{M_{b}} \frac{d \mathcal{N}}{d \mathcal{M}} d \mathcal{M} \int P(\Theta) \tau\left(>\mu_{\min }, z\right) d \Theta,
$$

where $\mathcal{R}(z)$ is the rate of events discussed in Sec. V and $d \mathcal{N} / d \mathcal{M}$ is the chirp mass function discussed in Sec. III (that to first order we assume it does not evolve with redshift). The integration mass limits in the integral are fixed to $M_{a}=5 \mathrm{M}_{\odot}$ and $M_{b}=50 \mathrm{M}_{\odot} . P(\Theta)$ is the probability distribution for the geometric factor, encoding all possible orientations of the detector and GW. For this term, we follow Ref. [27]. Finally, $\mu_{\min }$ is the minimum magnification required for a $\mathrm{GW}$ at redshift $z$, with chirp mass $\mathcal{M}$ and geometric factor $\Theta$ to be above the detection threshold of the experiment [see Eq. (2)]. We set a threshold in the signal-to-noise ratio of $\rho=8$ for noise properties similar to those in $\mathrm{O} 1$ and $\mathrm{O} 2$ runs in LIGO/Virgo (see

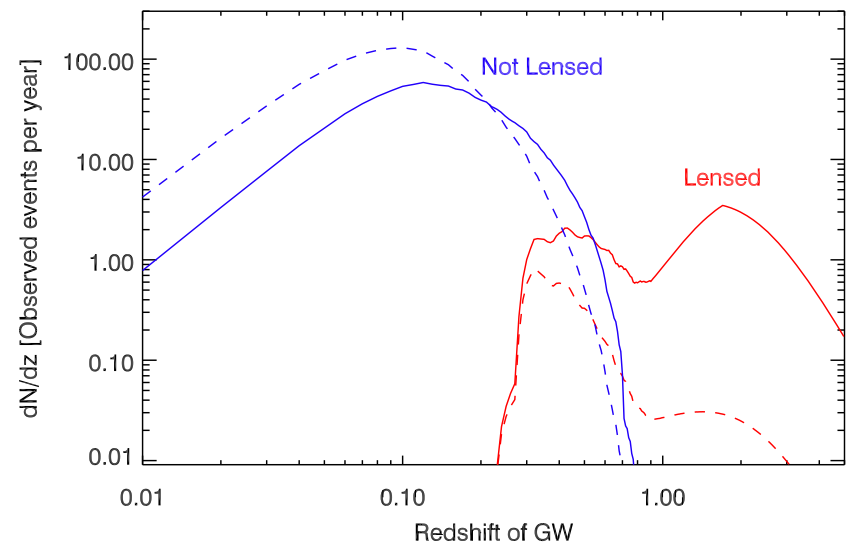

FIG. 4. Number of events above the detection threshold for a LIGO-like experiment with sensitivities comparable to those in the $\mathrm{O} 1$ and $\mathrm{O} 2$ runs. The dashed line is for the SFR model, and the solid line for the BDS model. Lensed events are given by the red curves. In the BDS model, most of the lensed events are all originating at redshifts $z_{s}>1$, while for the nonlensed events most of the observed events occur at $z<0.3$. The peak in lensed events at $z \approx 0.3-0.5$ are weakly lensed events.

Sec. II). By setting $\mu_{\min }=1$, we compute also the number of events that are not being lensed. The resulting number of observed events is shown in Fig. 4 as a function of the redshift of the GW and for the two rate models discussed in Sec. V. The dashed line (SFR model) shows results consistent with earlier estimates that predict that a small probability of lensed events is expected. The peak in lensed events at $z \approx 0.3$ corresponds to events with a mild magnification of $\approx 2$ for which the optical depth of lensing is higher (see Fig. 5). On the contrary, the BDS model (solid line) predicts similar numbers of lensed and notlensed events. The lensed events originate mostly at $z>1$. As in the SFR model, a peak of low magnification can be

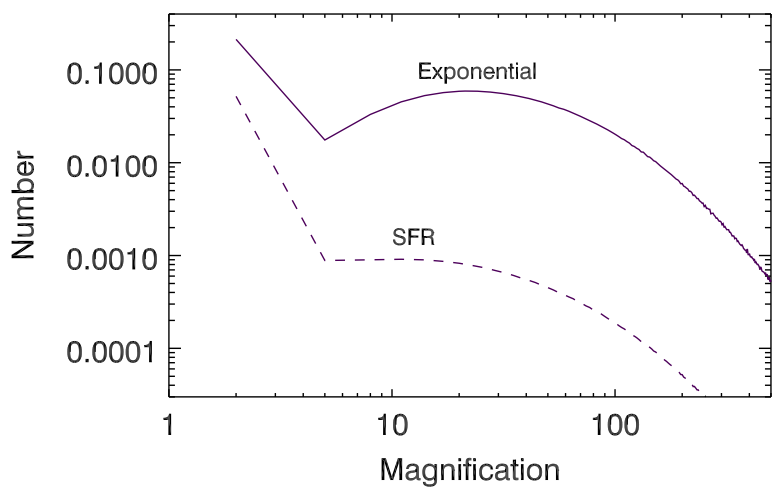

FIG. 5. Distribution of observed events as a function of the magnification. The peak at low magnifications are events that are being magnified by modest factors, including weak lensing events. For strongly lensed events $(\mu>5)$, the distribution peaks at magnification factors of $\approx 30$ for the exponential (BDS) model with a long tail toward larger magnification factors. This tail falls like $\approx \mu^{-3}$ for $\mu>200$. 


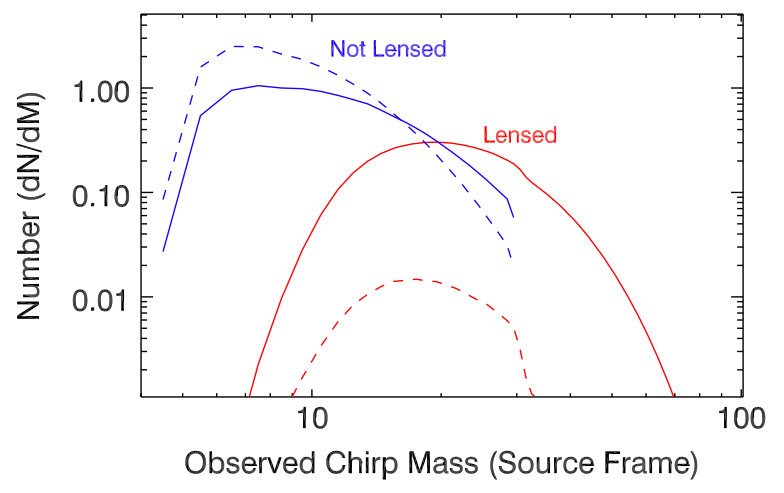

FIG. 6. Inferred observed chirp masses in the source frame assuming there is no magnification for the two types of models considered, SFR (dashed lines) and BDS (solid lines). Lensed events are shown in red and not-lensed events in blue. Note how in the BDS model (solid lines) the lensed events appear to have a higher chirp mass and how the observed chirp mass function appears bimodal.

appreciated also at $z \approx 0.3$. Figure 5 shows the distribution of magnifications that result in observed events. For the SFR model, most of the events are magnified by moderate factors, which makes them difficult to be recognized as lensed events. However, for the BDS model, most of the lensed events have magnifications of a few tens, and a nonnegligible fraction can have magnifications above 100.

Also interesting is the distribution of inferred chirp masses. Figure 6 shows the chirp masses inferred for the not-lensed and lensed events. As expected, the inferred masses for the not-lensed events resembles the underlying chirp mass (with a bias toward higher masses, because lower masses are less likely to exceed the detection threshold). However, for the lensed events (for which the mass has been inferred assuming the wrong magnification $\mu=1$ ), the peak of the distribution is around masses of 20 or 30 solar masses, in striking resemblance to the current observed LIGO masses. Reference [19] originally suggested that this mechanism was responsible for the apparently high chirp masses of most of the LIGO events.

\section{LENSING BY A MACROLENS PLUS MICROLENSES}

This section briefly reviews the lensing formalism for microlenses in a macrolens. This topic has been extensively covered in the literature [50-53]. Our model involves a macrolens and a population of microlenses. The microlenses include stellar microlenses (stars and remnants) and a population of PBHs. The PBHs account for a fraction $f$ of the total dark matter, where $f=1$ would imply that all dark matter is made of PBHs. Since in the range of $\approx 5-50 \mathrm{M}_{\odot}$ the fraction $f=1$ is already excluded by other observations, we consider only values consistent with current constraints. The surface mass density of dark matter is given by the convergence $\kappa$ of the macrolens. Then, the surface mass density of PBHs is simply $f \kappa$.

For the macrolens, we follow Ref. [16] and define the macrolens with just two parameters, the magnification factors in the radial and tangential directions, or $\mu_{r}$ and $\mu_{t}$, respectively. This is a valid approach for the macrolens, since we are dealing with very small regions of the sky. Without loss of generality, we assume that $\mu_{t} \gg \mu_{r}$ and that the main direction of the shear, $\gamma$, is oriented in the horizontal direction, that is, $\gamma_{2}=0$ and $\gamma=\sqrt{\gamma_{1}^{2}+\gamma_{2}^{2}}=\gamma_{1}$. Given $\mu_{t}$ and $\mu_{r}$, the corresponding values of $\kappa$ (convergence) and $\gamma$ (shear) can be found from the relation between $\kappa, \gamma, \mu_{r}$, and $\mu_{t}$. For a given choice of $\kappa$ and $\gamma$, the lens equation $[\vec{\beta}=\vec{\theta}-\vec{\alpha}(\vec{\theta})]$ of the macrolens can be expressed as

$$
\vec{\beta}=\vec{\theta}-\vec{\alpha}(\vec{\theta})=\left(\begin{array}{cc}
1-\kappa-\gamma_{1} & -\gamma_{2} \\
-\gamma_{2} & 1-\kappa+\gamma_{1}
\end{array}\right) \vec{\theta}
$$

where the positions in the source plane are given by the coordinates $\beta=\left(\beta_{x}, \beta_{y}\right)$ and the positions in the image plane are given by the coordinates $\theta=\left(\theta_{x}, \theta_{y}\right)$.

The lensing potential of the macrolens, $\psi$, is given by

$$
\psi=\frac{\kappa}{2}\left(\theta_{x}^{2}+\theta_{y}^{2}\right)+\frac{\gamma_{1}}{2}\left(\theta_{x}^{2}-\theta_{y}^{2}\right)-\gamma_{2} \theta_{x} \theta_{y},
$$

where we remind the reader that we adopt a reference system where $\gamma_{2}=0, \theta_{x}$ and $\theta_{y}$ are given in radians, and we ignore a constant additive term (i.e., the potential is identically zero at the origin of coordinates of $\theta$ ).

Since both the deflection field and lensing potential are linear with the addition of new masses, if a population of $N$ point masses are present, the deflection $\vec{\alpha}_{\mathrm{PS}}(\vec{\theta})$ and potential $\psi_{\mathrm{PS}}(\vec{\theta})$ from the distribution of point masses can be simply added to the above equations with

$$
\vec{\alpha}_{\mathrm{PS}}(\vec{\theta})=\sum_{i}^{N} \frac{4 G M_{i} D_{i}\left(z_{l}, z_{s}\right)}{c^{2}} \frac{\delta \vec{\theta}_{i}}{\left|\delta \vec{\theta}_{i}\right|^{2}}
$$

and,

$$
\psi_{\mathrm{PS}}(\vec{\theta})=\sum_{i}^{N} \frac{4 G M_{i} D_{i}\left(z_{l}, z_{s}\right)}{c^{2}} \ln \left(\left|\delta \vec{\theta}_{i}\right|\right),
$$

where $\delta \vec{\theta}_{i}=\vec{\theta}-\vec{\theta}_{i}$ is the distance to the point mass $i$ at $\vec{\theta}_{i}$ and with mass $M_{i}, D_{i}\left(z_{l}, z_{s}\right)$ is the geometric factor $D_{i}\left(z_{l}, z_{s}\right)=D_{l s}\left(z_{l}, z_{s}\right) /\left(D_{l}\left(z_{l}\right) D_{s}\left(z_{s}\right)\right)$ with $D_{l s}\left(z_{l}, z_{s}\right)$, $D_{l}\left(z_{l}\right)$, and $D_{s}\left(z_{s}\right)$ the angular diameter distances between the lens and the source, between the observer and the lens, and between the observer and the source, respectively.

A quantity of interest, that will be relevant in Secs. VIII and IX, is the effective optical depth $\tau_{\text {eff }}$ introduced by Ref. [17]: 


$$
\tau_{\text {eff }}=\left(4.2 \times 10^{-4}\right) \frac{\Sigma}{\mathrm{M}_{\odot} \mathrm{pc}^{-2}} \frac{\mu}{\mu_{r}},
$$

where the total magnification $(\mu)$ is the product of the tangential and radial magnifications (i.e., $\mu=\mu_{t} \times \mu_{r}$ ) and $\Sigma$ (expressed in units of $\mathrm{M}_{\odot} / \mathrm{pc}^{2}$ in the expression above) is the microlens surface mass density. When $\tau_{\text {eff }} \approx 1$, the saturation regime is reached. In this regime, caustics always overlap in the source plane, and any source moving across a field with $\tau_{\text {eff }}>1$ will be constantly experiencing microlensing $[17,35]$. Since typical values for $\Sigma\left(\mathrm{M}_{\odot} / \mathrm{pc}^{2}\right)$ range between a few to a few tens, and assuming a typical value for $\mu_{r} \sim 1$, it is clear from the expression above that the saturation regime is reached when the macrolens magnification is in the range of a few hundred to a few thousand. Similar values for the macrolens magnification have been observed already at the position of the microlensing events of the Icarus and Warhol high-redshift stars [54,55]. Given the fact that GW experiments have access to the entire sky at any given moment (as opposed to the aforementioned examples of Icarus and Warhol, where a significant luck factor had to be involved), events with similar, or even more extreme, macrolens magnifications are expected (see [35] for a detailed estimation of the probability of these events). In those scenarios, we expect microlensing to play a significant role.

Finally, the time delay is given by

$$
\Delta T=\frac{1+z_{l}}{c D\left(z_{l}, z_{s}\right)}\left[\frac{1}{2}|\vec{\theta}-\vec{\beta}|^{2}-\psi\right],
$$

where we have assumed that all point masses are at the same redshift in the lens plane, so the factors $D_{i}\left(z_{l}, z_{s}\right)$ are the same for all of them.

The time delay can be expressed in dimensionless units by rescaling both the angular positions and potential by the Einstein radius: $\theta_{E}^{2}=\left(4 G M / c^{2}\right) D\left(z_{l}, z_{s}\right)$. This redefinition of the time delay expression makes most sense when one is dealing with a single microlens, since in this case the Einstein radius can be defined without ambiguity, but, in general, one can still set the Einstein radius to any arbitrary mass, or scale, and still redefine the time delay equation:

$$
\Delta T=\frac{2 R_{s}\left(z_{l}\right)}{c}\left[\frac{1}{2}|\vec{x}-\vec{y}|^{2}-\tilde{\psi}\right],
$$

where $\vec{x}=\vec{\theta} / \theta_{E}, \vec{y}=\vec{\beta} / \theta_{E}, \tilde{\psi}=\psi / \theta_{E}^{2}$, and $R_{s}\left(z_{l}\right)$ is the redshifted Schwarzschild radius of the lens. The first term sets the scale of the time delay for a given lens mass: $2 R_{s}\left(z_{l}\right) / c=1.97 \times 10^{-5}\left(1+z_{l}\right)\left(M / \mathrm{M}_{\odot}\right)$ s. This simple scaling shows that, for a GW with frequencies $\nu \sim 100 \mathrm{~Hz}$, interference and diffraction effects are expected for isolated microlenses with masses $>500 /$ $\left(1+z_{l}\right) \mathrm{M}_{\odot}[8,11]$. Below this mass, the Schwarzschild radius of the lens is smaller than the wavelength of the GW (with $\nu \sim 100 \mathrm{~Hz}$ ), and diffraction effects are not important, resulting in the GW not seeing the microlens. However, as discussed in the section below, this minimum mass can be lowered substantially when the microlens is near a macrolens critical curve.

\section{A. The impact of microlenses on gravitational waves}

In earlier work, Ref. [17] discussed how a microlens with mass $M$ embedded in a region of a macrolens where the macrolens magnification is $\mu$ behaves as a microlens with an effective mass $\mu M$. Also, at larger magnifications, an area $A$ in the lens plane maps into a smaller area $A / \mu$ in the source plane. Hence, the density of microcaustics increases by a factor of $\mu$. Since the surface mass density of microlenses is roughly a constant fraction of the convergence (which is of the order of 1 near the critical curves of macrolenses), it is then easy to realize that a GW that is being magnified by a macrolens with a factor $\mu$ will inevitably be affected by microlensing (and its associated wave effects), if the magnification factor of the macrolens is large enough.

When the wavelength of a GW is comparable to the Schwarzschild radius of the microlens, one needs to consider wave optics instead of geometric optics. The magnification factor in wave optics is given by the diffraction integral (see, for instance, [56]):

$$
F(w, \beta)=A_{o} \frac{\nu}{2 \pi i} \int d^{2} \theta e^{i 2 \pi \nu \Delta T(\theta, \beta)},
$$

where $\nu$ is the frequency of the GW in hertz and the normalization $A_{o}$ guarantees that at very high frequencies one recovers the geometric optics magnification when averaged over a frequency range. $\Delta T$ is the time delay between lensed images expressed in seconds. The total magnification $\mu$ and phase shift $\phi$ are given, respectively, by (see, for instance, [25])

$$
\begin{gathered}
\mu=|F(w, \beta)|^{2}, \\
\phi=-i \ln \left(\frac{F(w, \beta)}{|F(w, \beta)|}\right) .
\end{gathered}
$$

For isolated microlenses and simple macrolenses, analytic solutions can be found for the magnification. The magnification depends on the frequency and that at low frequencies the magnification tends to 1 (i.e., the microlens has a negligible effect over the $\mathrm{GW}$ ). In realistic situations, where a GW is strongly lensed, it may intersect not only one microlens but different microlenses. In this case, the integral in Eq. (11) can be solved numerically as described in Refs. $[9,16,57]$, basically by inverse Fourier transforming the probability function of the time delays, $F(\Delta t)$. This numerical method can be applied to any situation, independently of its complexity, provided one computes the 
time delay surface with enough resolution to resolve the peaks in $F(t)$. The case of a pair of overlapping microlenses was studied in Ref. [35]. In this paper, we study the more realistic situation where a distribution of microlenses intersects the GW. The probability that a $\mathrm{GW}$ intersects a microlens and suffers a distortion in its waveform depends on the density of microlenses and the size of the caustic region. As discussed at the beginning of this section, this probability increases with $\mu$. Hence, GWs with large magnification factors are more likely to suffer interference than GWs with modest magnification factors. We consider two scenarios, the first one where the macrolens magnification is 30 and a second scenario where the macrolens magnification is 5 times larger (that is, 150). As shown in Sec. VI, the most likely magnification factor for models such as the BDS one is $\mu \approx 30$. Despite the rapid decline of the optical depth with magnification, observed events with $\mu=150$ are only $\approx 5$ times less likely than observed events with $\mu=30$ (for the BDS model), since the reduction in lensing probability is partially compensated by the increase in the accessible volume. For the SFR model, the rate of probabilities is similar, but the probability of observing events with $\mu=30$ is 2 orders of magnitude smaller, so events like the ones described in this work would be observed only after the sensitivity of the detectors improves.

\section{PRIMORDIAL BLACK HOLES AS DARK MATTER}

In the previous sections, we discussed the lensing probability for the SFR and the BDS models. In both cases, lensing events are expected to take place, although, in the case of the BDS model, these events are $\approx 2$ orders of magnitude more likely, and should have already been observed by LIGO. As mentioned above, the BDS model is appealing, because it predicts a bimodal mass function in the observed chirp mass, consistent with the observed distribution of chirp masses, where the data cluster preferably around two peaks, one at masses $\approx 15 \mathrm{M}_{\odot}$ and one at $\approx 30 \mathrm{M}_{\odot}$. On the contrary, if the BDS model is correct, lensed events with inferred large chirp masses should come in pairs with time delays between a few hours to several days and from the same location in the sky. To date, no clear multiply lensed GW has been observed [58,59], although several candidates have been proposed [60]. More recently, two events during the $\mathrm{O} 3$ run were observed on August 28, 2019, which are separated in time by $\approx 19$ min and seem to originate from similar luminosity distances and positions in the sky. These positions are separated by only $\approx 10^{\circ}$. To first order, the probability of having two events so close in time and space is comparable to the probability of having a strongly lensed event. However, based on the sky location of these two events, Ref. [61] concluded that the two events cannot be interpreted as a pair of strongly lensed images. As shown below, if GWs are strongly lensed and microlenses are present, the observed strains can be perturbed, especially at the largest frequencies. The impact of this perturbation on the estimation of the parameters of the GW is something that needs to be addressed carefully. Moreover, repeated events arriving at different times (due to time delay lag) may be confused with a bursting signal. Searches for unmodeled, or GW burst, signals in GW have resulted in no burst detections so far (note that burst searches can also return nonbursting signals [62]), although these searches restricted themselves to Gaussian pulses, sine-Gaussian wavelets, and white-noise bursts [63]. Interestingly, if these burst searches resulted in any result, the sky localization of the signal would be less constrained, given a reduced precision in arrival times [64].

In this section, we assume that strongly lensed GWs have been observed already (as suggested by the BDS model) or that they will be observed in the near future and investigate the possibility of constraining the abundance of PBHs. We take advantage of the magnifying power of the macrolens hosting the PBHs, which increase the concentration of caustics in the source plane, resulting in complex time delay distributions and interference patterns of the lensed GWs. We create a set of simulations where we vary the fraction of dark matter contained in PBHs and compute the distortion in the magnification as a function of the frequency for different positions of the GWs in the source plane. Sampling the source plane allows us to perform a statistical analysis, where we estimate the probability that a $\mathrm{GW}$ is distorted by some fraction at a given frequency. The simulations involve three components, a macrolens (galaxy or cluster), stellar microlenses from the macrolens, and PBHs that constitute a fraction $f$ of the dark matter of the macrolens. By construction, $f<1$, and we consider fractions $f$ that are consistent with current upper limits. We neglect projection effects and consider only deflectors that are in the macrolens plane. Projection effects are expected to contribute at the percent level, since the adopted value for the convergence of the macrolens is of the order of 1. For the macrolens, we follow Ref. [16] and model the macrolens with just two parameters, the convergence $\kappa$ and the shear $\gamma$. The specific values of $\kappa$ and $\gamma$ are derived from predetermined values of the magnification in the tangential $\left(\mu_{t}\right)$ and radial $\left(\mu_{r}\right)$ directions. We consider two scenarios for the magnification $\mu$ of the macrolens. In the first scenario, we adopt a relatively modest magnification of 30, resulting from the product of a tangential magnification of 10 and a radial magnification of 3 , that is, $\mu=\mu_{t} \mu_{r}=10 \times 3=30$. In the second scenario, we consider a more extreme value of the magnification $\mu=\mu_{t} \mu_{r}=50 \times 3=150$. The two scenarios can be considered as two nearby positions in the image (or source) plane, where the second scenario corresponds to the position that is closest to the critical curve (or caustic). For typical macrolenses with the mass of a massive galaxy, the magnification can change from 30 to 150 in image 
plane distances smaller than one arcsec. In addition to the two considered values for the macrolens magnification, we also consider the two possible parities of the macroimages. A source located near a caustic produces typically two bright macroimages (in general, additional macroimages are also formed, but with significantly less magnification and time delays much larger than the duration of a GW event, so they can be neglected). One of the two macroimages has positive parity (i.e., it has the same orientation of the orbital momentum as the source), and the other has negative parity (i.e., it resembles a mirror image of the source). Other than the change in symmetry, in the presence of microlenses in the lens plane, the parity of the macroimage is important when dealing with very small sources. As shown in earlier work (see, for instance, [16] for a discussion in the context of GWs), macroimages of very small sources with negative parity are significantly more likely to have smaller magnifications than the corresponding macroimage with positive parity, if microlenses intersect the line of sight. The effect is even more dramatic for GWs, since the microlenses introduce a time delay that can be more pronounced between microimages forming around a macroimage with negative parity. The time delay between microimages results in constructive and destructive interference patterns affecting the GW. This effect depends on the frequency of the GW and can resemble (and could, in principle, be confused with) spin misalignment. However, as opposed to the case of spin misalignment, when microlensing is taking place and the time delay is larger than the inverse of the frequency of the wave, the last bright peak before the merger can be observed multiple times (see, for instance, Fig. 2 in Ref. [65]). As a function of time, the observed signal would resemble echoes (or bursts), since the wave carrying the last bright peak arrives multiple times. The first echo will be affected by interference more than the last one, which will be almost unaffected by interference (assuming the ringdown phase is shorter than the time delay between the last and second-to-last echoes).

Since the results depend on the parity of the macroimage, this allows one to confirm that a GW is being strongly lensed, since one would expect (on average) that the image with positive parity arrives first and a few hours (or days) later the second image with negative parity would arrive. This second image is expected to show stronger distortions with frequency, on average, if microlensing is involved. If $\mu$ is sufficiently large ( $\mu>$ larger than a few hundred), this type of behavior is always expected, since, even in the absence of PBHs, microlenses from the intergalactic or intracluster medium can saturate the source plane with microcaustics. Events with extreme magnification can be recognized, because they may originate at very high redshifts $(z>2)$ and be misinterpreted (if lensing is ignored) as events with unusually high masses $m_{1}$ and $m_{2}$, that could even fall within the mass gap above $50 \mathrm{M}_{\odot}$.

In all simulations, we include the stellar component using the same model described in Ref. [16]. For the two macrolens magnifications considered (30 and 150), we simulate a circular area in the image plane of 3 milliarcsec (mas) in diameter but consider only the central horizontal rectangular area of $3 \times 0.5 \mathrm{mas}^{2}$. The resolution is 100 nanoarcsec per pixel, sufficient to properly resolve the caustic regions around microlenses with stellar masses. The area in the source plane is reduced by the corresponding magnification factor of the macrolens. The contribution of the stellar component is modest, and only the (rare) most massive stars and remnants are expected to produce a significant effect on the GWs. The contribution to $\kappa$ of the stars and remnants is typically at the $\sim 1 \%$ level, and most of the mass is contained in objects with masses $\sim 1 \mathrm{M}_{\odot}$ or less. At masses above $\sim 5 \mathrm{M}_{\odot}$, where the interference effects are more prominent, stars and remnants contribute to the total mass a fraction much smaller than the contribution of the PBHs considered in this work. Because of this, the impact of the particular modeling of the stellar component is expected to be minor in our conclusions. Nevertheless, they are included assuming a surface mass density of $11.7 \mathrm{M}_{\odot} \mathrm{pc}^{-2}$, which is the same value used in Ref. [16] and a typical value found around the critical curves of macrolenses for background sources with $z>1[54,66]$.

For the mass function of the PBHs, we assume that their mass function is not necessarily the same as the one assumed for the sources (see Sec. III). Instead, we assume two different models. First, we consider a Gaussian distribution. This is a simple extension to the monochromatic model with a fixed mass that allows for a broadening of the mass function. A log-normal mass function could be used instead, but we expect negligible differences in our conclusions, provided both Gaussian and log-normal have comparable dispersion. Similar models have been considered in the past to describe a possible population of PBHs with masses comparable to those found by LIGO. This type of model is already constrained by current observations, which rule out fractions larger than $f \approx 0.1$. In this work, we consider fractions that do not violate these limits. In particular, we consider values of $f=0.033,0.01$, and 0.003 , that is, $3.3 \%, 1 \%$, and $0.3 \%$ the amount of dark matter, respectively. We refer to these three models as Gaussian models, and more specific details about these models are provided below. Interestingly, a fraction of $\approx 1 \%$ may be sufficient to explain the rate of mergers observed by LIGO [7,67-69].

As a second model for the population of $\mathrm{PBHs}$, we consider a much broader mass function, which is still consistent with current constraints [70] but can allow for a larger fraction of the dark matter to be explained by PBHs. This is possible as long as the PBHs span a wide range of masses and the fraction of mass contained in each range does not violate current limits [70-72]. We model this second model as a power law between $M_{\min }$ and $M_{\max }$ and refer to this type of model as the power-law model: 


$$
f=\frac{\int_{M_{\min }}^{M_{\max }} d M M \Psi(M)}{\kappa * \Sigma_{\text {crit }}},
$$

where $\kappa$ is the convergence, $\Sigma_{\text {crit }}$ is the critical surface mass density, and $\Psi(M)$ is the mass function of the PBH per unit area. We assume that it follows a power law: $\Psi(M)=d N / d M \propto M^{\gamma_{p}-1}$. The index $\gamma_{p}$ is a free parameter, and we consider two values, $\gamma_{p}=0$ and $\gamma_{p}=1$. When $\gamma_{p}=0, f$ is a constant as a function of the mass. When $\gamma_{p}=1$, the number of PBHs per mass interval is constant, and, hence, more massive PBHs contribute more to $f$ than lower-mass PBHs.

This mass function was also considered in Ref. [73], that argues that "a mass function of this form arises naturally if the PBHs form from scale-invariant density fluctuations or from the collapse of cosmic strings." For the limits of the integral, we consider $M_{\min }=5 \mathrm{M}_{\odot}$ and $M_{\max }=50 \mathrm{M}_{\odot}$. This range is justified, because wave effects are not very important for masses below $5 \mathrm{M}_{\odot}$. On the other extreme, above $50 \mathrm{M}_{\odot}$, the fraction of PBH is heavily constrained. Outside this mass range, there may still be $\mathrm{PBHs}$ that contribute to $f$. To account for this possibility, we consider values of $f$ smaller than 1 but still larger than the values of $f$ adopted for the Gaussian model. Note, however, that in Ref. [74] the authors propose a mass function that (they claim) is consistent with current microlensing observations and can explain all dark matter as PBHs. In particular, in this model the mass function of PBHs is multimodal, with the peaks of the mass function concentrated around $10^{-6}, 1$, 30, and $10^{6} \mathrm{M}_{\odot}$.

For a given choice of the mass function, the number of PBHs per unit area inserted in the simulation is determined by the specific values of $f$ and $\kappa$. The convergence is determined by the values of $\mu_{t}$ and $\mu_{r}$ as described in Ref. [17].

\section{A. Gaussian model}

First, we consider the case of the Gaussian model. We assume a Gaussian mass function for the PBHs with mean $30 \mathrm{M}_{\odot}$ and dispersion $5 \mathrm{M}_{\odot}$. This model is inspired by the high-mass peak found in the bimodal mass function in Ref. [31]. Since the dispersion is relatively small, the results presented in this section would be close to those obtained with a monochromatic mass function with masses equal to the mean value of the Gaussian. In Fig. 7, we show the magnification in the image plane for the simulations in the Gaussian model case and for the case with $\mu=150$. The magnification diverges at the critical curves (white lines). The top three panels show the magnification on the side of the image plane where the parity is positive and for the three values of $f$ that are consistent with current constraints [75]. From top to bottom, $f=0.3 \%, f=1 \%$, and $f=3.3 \%$. The bottom three panels are the corresponding magnification maps in the image plane for the side of the
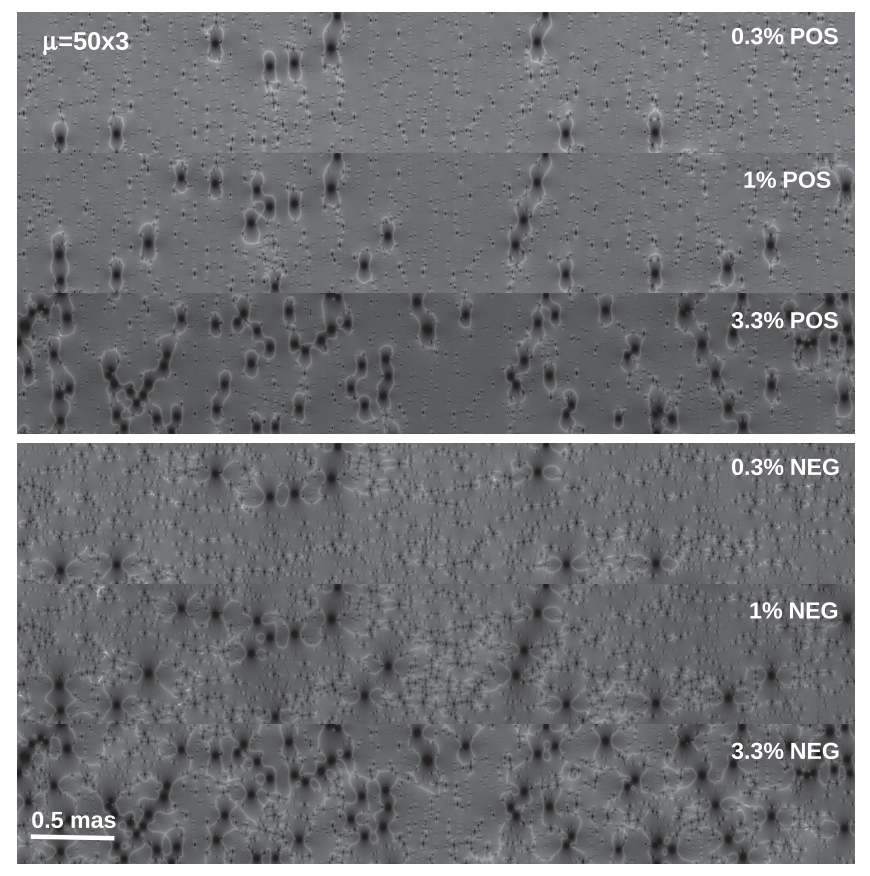

FIG. 7. Magnification in the image plane showing the critical curves (white lines) for a macrolens magnification $\mu=\mu_{t} \mu_{r}=50 \times 3=150$. The top three panels correspond to three simulations (with different contributions to the dark matter content) on the side of the image plane with positive parity. The bottom panels are the corresponding simulations at the same macrolens magnification in the side with negative parity. The relatively small critical curves are due to stars in the macromodel, and the larger critical curves correspond to the PBHs.

image plane with negative parity and the same values of $f$. In both cases (positive and negative parities), the dark-gray areas have magnification factors of a few. The largest critical curves are around the PBHs. Smaller critical curves correspond to stars and remnants in the macrolens. Note how the orientation (and shape) of the critical curves depends on the sign of the parity. The case with $\mu=30$ is not shown but would look similar with the exception that the critical curves around the microlenses would be smaller by a factor of $\sqrt{150 / 30}$. By using the lens equation, we map the magnification in the image plane into the magnification in the source plane, where the critical curves map into caustics. Since the image plane gets compressed by a factor of $\mu$ in the source plane, it is often the case that, at large macrolens magnifications, the caustics overlap. In this scenario, images can form around critical curves that are separated by relatively large distances in the image plane. For these images, the geometric time delay can be larger than the time delay that would be observed if the caustics did not overlap, resulting in interference effects at even lower frequencies. This overlapping effect in the source plane is shown in Fig. 8, where the three top panels are for the case with positive parity and the three panels in the bottom are for the case with negative parity. In this case, the 

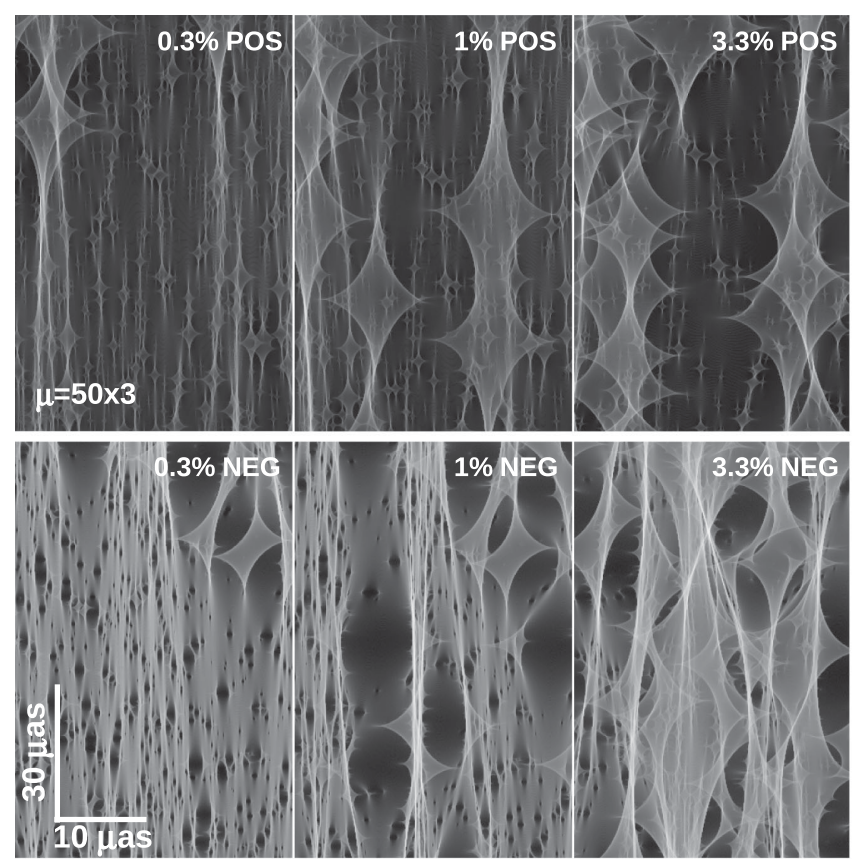

FIG. 8. Corresponding caustics in the subregion used to compute the time delay statistics. The images have been compressed by a factor of $\approx 2$ in the vertical direction in order to show a larger area. The maximum magnification is similar for both parities, but the minimum magnification is significantly smaller in the case of negative parity.

caustics look very different depending on the sign of the parity. On the side with positive parity, the minimum magnification (dark-gray areas) is of the order of the macrolens magnification (i.e., $\mu_{\min } \approx 150$ ), while on the side with negative parity the dark-gray areas have a magnification of just a few. For both parities, at fractions $f$ above a few percent and at this macrolens magnification, caustics start to overlap across the source plane. At even larger macrolens magnification factors, the overlap start to take place at smaller values of $f$.

We use the simulated source planes to estimate the probability that a GW is distorted by some amount at a given frequency. We place $300 \mathrm{GWs}$ at random positions in the source plane shown in Fig. 8 and compute the magnification of the GW (relative to the macrolens magnification that does not depend on the frequency) as a function of the frequency following Ref. [16] for each position. Each of the 300 random positions [ $\beta$ in Eq. (11)] results in a distortion curve of relative magnification as a function of the frequency [ $w$ in Eq. (11)]. In Fig. 9, we show the first 50 curves, out of the 300 examples for a particular choice of the PBH (that is described below in Sec. VIII B). Let us remind the reader that these curves represent the relative magnification with respect to the macromodel and that the macromodel magnification is expected to be independent of the frequency. For clarity purposes, we highlight the first three curves as colored solid

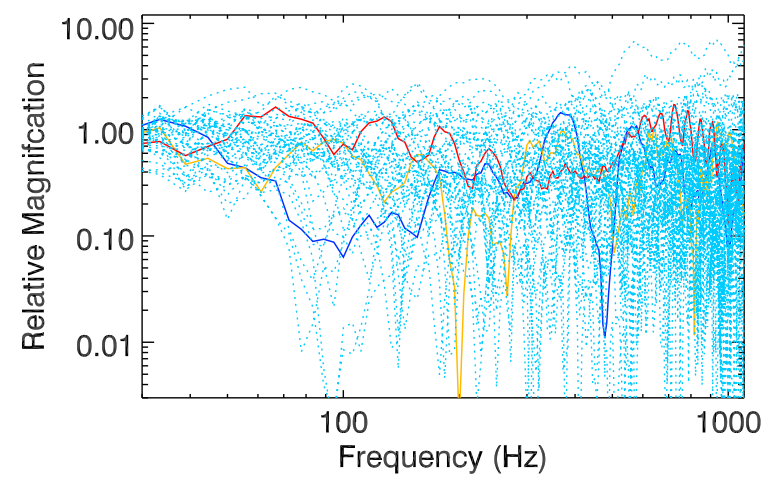

FIG. 9. Examples of the relative magnification induced by microlensing as a function of the GW frequency for the case of negative parity and the power-law model with $\gamma=1$. The colored lines are the first three curves from the randomly selected positions. The light-blue dotted lines show the first 50 curves from the random positions. Note how at low frequencies, $\nu<60 \mathrm{~Hz}$, all simulated cases show relatively small distortions in the relative magnification.

lines (blue, orange, and red). As shown in the figure, some of the curves show significant deviations from unity at frequencies as low as $100 \mathrm{~Hz}$. For instance, the dark-blue solid curve shows a large decrement between $\approx 80$ and $\approx 150 \mathrm{~Hz}$. The same curve shows an increase in the magnification (relative to the macrolens) at $\approx 400 \mathrm{~Hz}$, followed by a large reduction in the relative magnification at $\approx 500 \mathrm{~Hz}$. All curves tend to 1 at lower frequencies, as expected, since at these frequencies the GWs become insensitive to microlenses with the masses considered in this work. The corresponding distorted strains are discussed in Sec. IX below. Note that this plot is in frequency space, and matched filters are usually smooth in this space. At the positions of spikes in the power spectral density of the detector (like the harmonics of the detector which happen at well-known frequencies), the features produced by interference will be harder to detect.

With the 300 magnification curves, we compute the probability that the microlensing distortion of a $\mathrm{GW}$ is larger than some amount as a function of the frequency. For this goal, we compute the envelopes of the curves at different percentage levels $(50 \%, 90 \%$, and $97 \%)$ and as a function of the frequency. The envelopes at the $50 \%$ level indicate the minimum distortion (at a given frequency) in the magnification of $50 \%$ of the curves; that is, one in two GWs will have a distortion at least as large as the one shown by the envelope at $50 \%$ at that frequency. The envelopes at the $90 \%$ level indicate the minimum distortion in the magnification of $10 \%$ of the cases. That is, one in ten GWs will have a distortion in the magnification at a given frequency that is at least as large as the one shown by the envelope. Similarly, for the envelope at 97\%, approximately one in 30 GWs will have a distortion at a given frequency at least as large as the one shown by the envelope. Figure 10 shows the envelopes (or probabilities) 


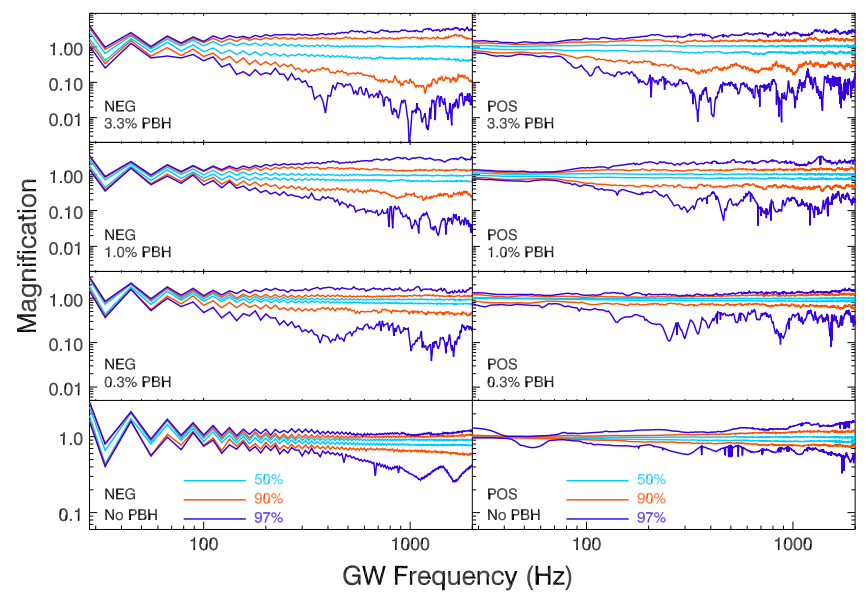

FIG. 10. Probability of magnification as a function of the GW frequency for a macrolens with magnification $\mu=\mu_{t} \mu_{r}=$ $10 \times 3=30$. The light-blue, red, and dark-blue curves bracket the regions with the $50 \%, 90 \%$, and $97 \%$, respectively, of the distortion at a given frequency. The side with negative parity shows, in general, a stronger distortion.

for the case with macrolens magnification $\mu=30$. The cases where the macrolens parity is negative are shown in the left column, while the cases where the parity is positive are in the right column. From top to bottom, we show the cases with $f=0.033,0.01$, and 0.003 and $f=0$. The case with $f=0$ has only the stellar microlenses (stars and remnants with a surface mass density of $11.7 \mathrm{M}_{\odot} / \mathrm{pc}^{2}$ following the model of Ref. [76]) and no PBHs. The serrated pattern observed in the envelopes with negative parity at low frequencies is an artifact due to numerical limitations (and related to the limited size of the simulation) when solving numerically the integral in Eq. (11). The envelope at these low frequencies is supposed to be a smooth version of the curves. As expected, the distortions are larger at higher frequencies. If one considers the case with $f=0.01$ (or $1 \%$ of the dark matter in the form of PBHs) and positive parity (i.e., right column, second panel starting from the top) at $500 \mathrm{~Hz}$, then $10 \%$ (90\% envelope) of the strongly lensed GWs with $\mu=30$ are expected to show a strong distortion in the magnification where destructive interference demagnifies the GW by a factor of $\approx 3$ (relative magnification of $\approx 0.3$ ). Since the strain goes as the square root of the magnification, this translates into a reduction of a factor of $\approx 2$ in the amplitude of the strain at $500 \mathrm{~Hz}$.

If the fraction of dark matter in PBHs increases, at $f=3.3 \%$, destructive interference with even greater intensity is evident at frequencies as low as $\approx 200 \mathrm{~Hz}$, well within the range of detectors such as LIGO. The distortion is even more apparent for the counterimages with negative parity (that we remind the reader should arrive between a few hours to a few days after the GW with positive parity) as shown by the left column. In this case, at least one in ten GWs with magnification above $\mu=30$ should show a

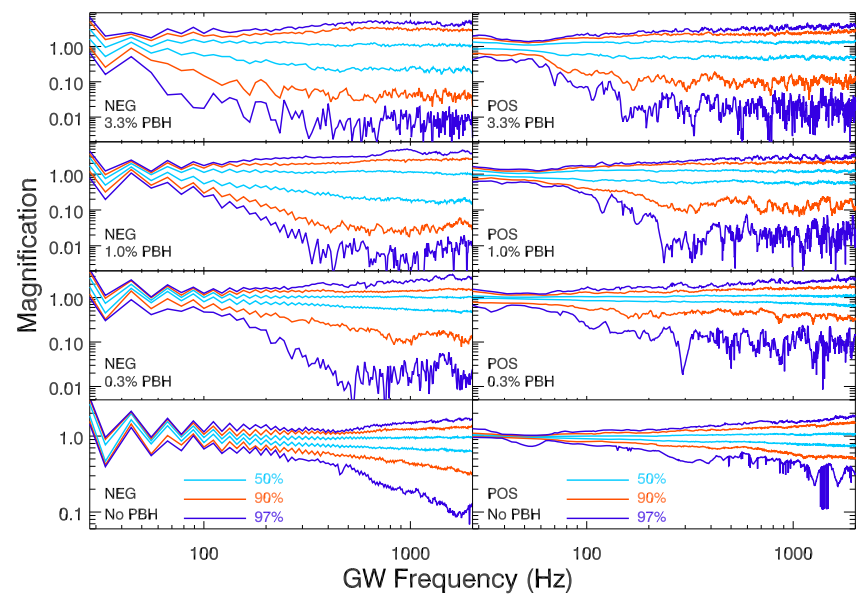

FIG. 11. As in Fig. 10 but for a position in the image plane where the macrolens has magnification $\mu=\mu_{t} \mu_{r}=50 \times 3=$ 150. In this case, the larger macromodel magnification results in a stronger effect for the same number of PBHs.

reduction in the strain by a factor of $\approx 2$ at frequencies of $200 \mathrm{~Hz}$. We should note that the envelope accounts only for the most extreme distortion at that frequency. This means that the event mentioned above with a reduction of a factor of $\approx 2$ at frequencies of $200 \mathrm{~Hz}$ at higher frequencies may show a smaller distortion (or even no distortion at all in a given frequency range).

The above result shows the potential of strongly lensed GWs to probe the abundance of PBHs, but it requires observing several events in order for one of them to show significant distortions in the observed strain. However, if we focus on events with even larger macrolens magnification factors, the caustics will overlap even more, and individual microlenses will behave as microlenses with larger masses (with an effective mass that scales as $\mu M$ ). In Fig. 11, we show the case for a macrolens magnification $\mu=150$. Note that, although the probability of an event with $\mu>150$ is $5^{2}$ times smaller than the probability with $\mu>30$, the probability of observing such an event is actually only a few times smaller, since at $\mu>150$ one is probing a larger volume than at $\mu>30$ (see, for instance, Fig. 5). For lensed events with $\mu>150$, and if one considers the case where $1 \%$ of the dark matter is in the form of PBHs, the $50 \%$ envelope is already at magnification factors 0.3 at frequencies of $\approx 300 \mathrm{~Hz}$. That is, a reduction in the strain of a factor of $\approx 2$ at $300 \mathrm{~Hz}$ is expected in $50 \%$ of the lensed events with $\mu>150$. One in ten of these events (90\% envelope) would have a similar reduction of a factor of 2 in the strain at frequencies as low as $\approx 130 \mathrm{~Hz}$. The same fraction would show reductions of a factor of 5 in the strain at $400 \mathrm{~Hz}$.

\section{B. Power-law model}

The second scenario we consider is the case where the mass function of PBHs between $5 \mathrm{M}_{\odot}$ and $50 \mathrm{M}_{\odot}$ is 
described by a power law. Given the current constraints, PBHs can make up to $\approx 10 \%$ of the dark matter [70]. If one relaxes the constraints at very low masses (asteroids) from PBH evaporation into gamma rays and at masses above $50 \mathrm{M}_{\odot}$ from the cosmic microwave background (Planck), PBHs can make up to $100 \%$ of the dark matter. In Ref. [73], the authors explicitly consider extended mass functions with power laws similar to the ones considered in this work and find that, in the mass regime we are interested in, a fraction as high as $10 \%$ is consistent with current constraints. We consider this value of $f=0.1$ in the case of the power law and make a set of simulations similar to the ones in Sec. VIII A $(\mu=30, \mu=150$, positive, and negative parities) for the two power laws considered, $\gamma_{p}=0$ and $\gamma_{p}=1$.

The result is shown in Fig. 12. In this case, and for simplicity, we consider only two envelopes at 50\% and $90 \%$. Not surprisingly, since there are more PBHs per unit area, the effect is larger than for the Gaussian model described in Sec. VIII A. As a general trend, we observe again that images with negative parity are more likely to show interference effects. The differences between the two power-law models is relatively small. The difference is also small when comparing the $\mu=30$ and $\mu=150$ cases, except at low frequencies, where interference effects in the case with $\mu=150$ are more evident. This can be explained because at fractions $f=0.1$, and for magnifications $\mu>30$, the saturation level is reached in the source plane (see [17] for a discussion of the saturation level), where microcaustics overlap over the entire source plane, and the statistical properties of the magnification are very similar at $\mu=30$ and $\mu=150$. Only at smaller fractions $f$ does the

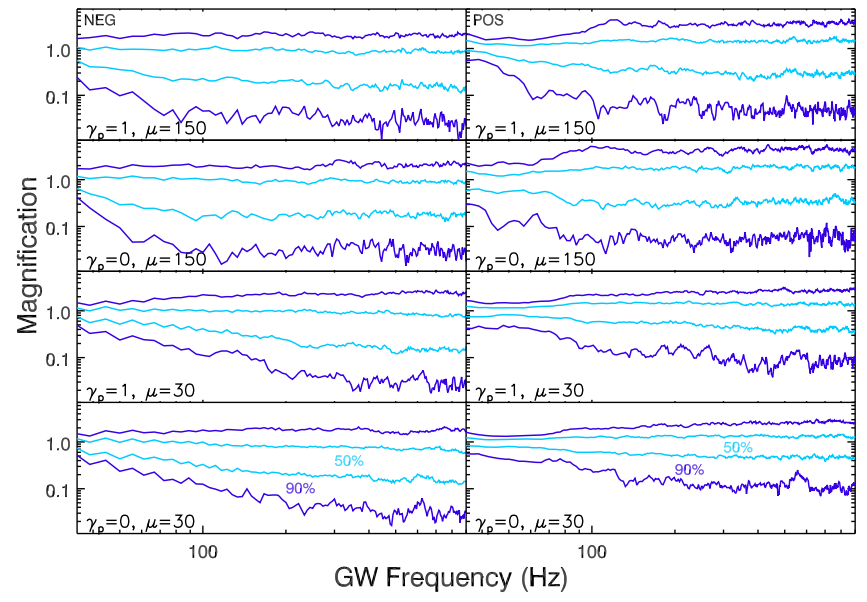

FIG. 12. Envelopes at $50 \%$ and $90 \%$ showing the probability of distortion as a function of the frequency for the two power-law models $\gamma_{p}=0$ and $\gamma_{p}=1$, the two macromodel magnifications considered $(\mu=30$ and $\mu=150)$, and for a fixed fraction $f=10 \%$. The case with stronger macromodel magnification, $\mu=150$, shows significant departures in $10 \%$ of the realizations (dark-blue curves) above $\approx 50 \mathrm{~Hz}$ for the power-law model with $\gamma_{p}=1$ and above $\approx 80 \mathrm{~Hz}$ for the model with $\gamma_{p}=0$.

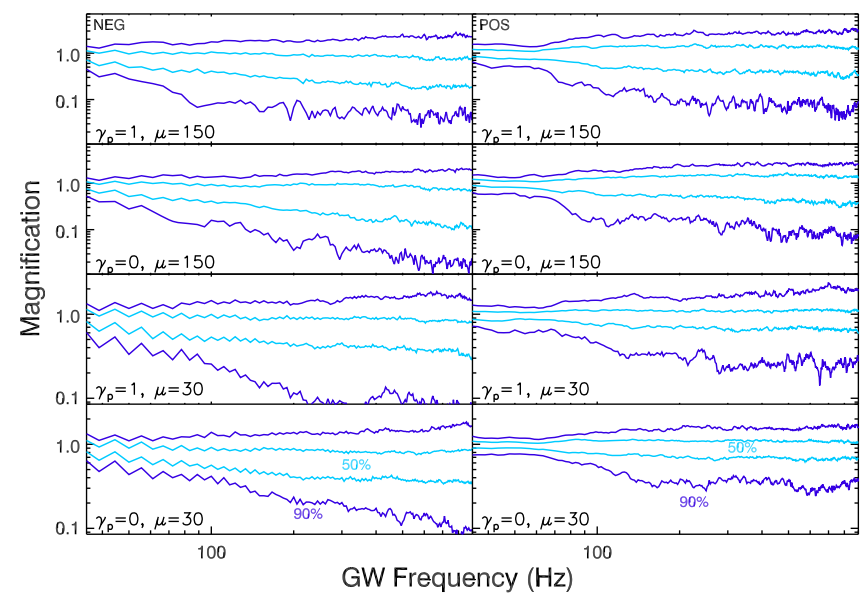

FIG. 13. As in Fig. 12 but for a smaller fraction $f=3.3 \%$. Note that the scale in the $y$ axis is different than in Fig. 12. As expected, the distortion is smaller in these cases than in the cases shown in Fig. 12.

probability of interference become sensitive to the macrolens magnification for magnification factors $\mu>30$, as shown in Fig. 13. For smaller magnification factors, the saturation regime is not reached, and the probability of intersecting a caustic becomes sensitive to the masses of the microlenses.

An interesting trend is observed in all cases with negative parity (left column in Figs. 12 and 13), where in more than $50 \%$ of the cases the magnification decreases with an increasing frequency. In the $90 \%$ envelope, for the images with both positive and negative parity, GWs are heavily suppressed above $100 \mathrm{~Hz}$. For the images with negative parity, this happens at even lower frequencies. In particular, $10 \%$ of the GWs with magnification $\mu=150$ would be heavily suppressed below $60 \mathrm{~Hz}$. Since most of the signal to noise in the events observed by LIGO is concentrated below $100 \mathrm{~Hz}$, this means that these events would basically be unobserved. In $50 \%$ of the cases, at $\mu=150$ the suppression factor is typically a factor $>2$ at frequencies $>100 \mathrm{~Hz}$ for images with negative parity and a bit smaller than 2 for the images with positive parity and at the same frequencies.

\section{DISCUSSION AND CONCLUSIONS}

The results in the previous sections show how, if a fraction of the dark matter is composed of microlenses with masses above a few $\mathrm{M}_{\odot}$, a strongly lensed GW may reveal this population of microlenses through interference effects. In general, the distortion is larger the larger is the fraction of dark matter in the form of compact microlenses, but at sufficiently high magnifications, the saturation level is reached [when $\tau_{\text {eff }} \approx 1$; see Eq. (8)] and the microcaustics overlap in the source plane. Once this saturation level is reached, the results are less sensitive to the number and masses of the microlenses, but the point where the 


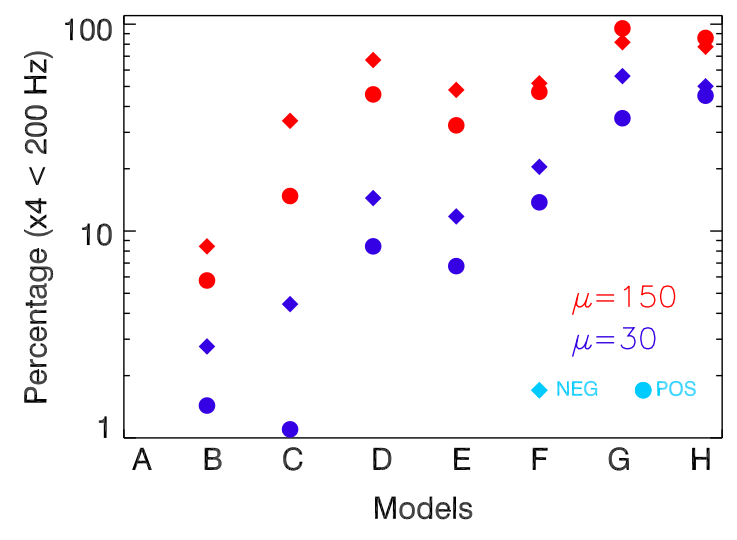

FIG. 14. Probability of having a distortion in the magnification larger than a factor of 4 at frequencies below $200 \mathrm{~Hz}$. The circles are for images with positive parity, while the diamonds are for images with negative parity. Blue symbols are for macroimages with magnification 30, and red symbols are for macroimages with magnification 150. Model A is the one with no PBHs (i.e., only stars and remnants with $\Sigma=11.7 \mathrm{M}_{\odot} \mathrm{pc}^{-2}$ ). For model A, the percentage falls below $1 \%$ and is not shown. Models B, C, and D are the three Gaussian models with $f=0.3 \%, 1 \%$, and $3.3 \%$, respectively. Models $\mathrm{E}$ and $\mathrm{F}$ are power-law models with $f=$ $3.3 \%$ and with $\gamma=0$ (E) and $\gamma=1(\mathrm{~F})$. Models $\mathrm{G}$ and $\mathrm{H}$ are power-law models with $f=10 \%$ and with $\gamma=0$ (G) and $\gamma=1(\mathrm{H})$.

saturation level is reached depends on the surface mass density of microlenses. Since the critical surface mass density for typical redshifts of lenses and background sources is $\Sigma_{\text {crit }} \approx 2000 \mathrm{M}_{\odot} \mathrm{pc}^{-2}$ and near the critical curves $\kappa \approx 1$, if the fraction of dark matter in microlenses is $\approx 3 \%$, then $\tau_{\text {eff }} \approx 1$ when the magnification of the macrolens is $\mu \sim 100$ (assuming the radial magnification factor is $\approx 2$ ). At smaller magnification factors, such as $\mu \approx 30$, one expects it to be more sensitive to the particular mass function of microlenses but at the expense of having fewer GWs with a significant distortion in the magnification, since the area in the source plane which is not close to a microcaustic is larger in this case.

In Sec. VIII B, the envelope curves shown in Figs. 10-12 account for the probability of distortion of a certain magnitude at a given frequency. Alternatively, it is interesting to ask the question of what the probability is of having a magnification of at least a factor of $\mu^{*}$ (a strain distortion of a factor of $\sqrt{\mu^{*}}$ ) below some frequency $\nu^{*}$. In Fig. 14, we show the case for $\mu^{*}=4$ and $\nu^{*}=200 \mathrm{~Hz}$. Below this frequency, one can expect that a distortion in the strain of a factor $>2$ at some frequency $\nu<200 \mathrm{~Hz}$ could be picked by optimally filtering the raw data. Each curve represents all the models discussed in Sec. VIII B. The Gaussian models are one the left side of the $x$ axis, while the power-law models are on the right side. Dashed lines are for macroimages with negative curvature, and solid lines are for macroimages with positive curvature. In general, and as expected, it can be appreciated how models with a larger fraction $f$ are more likely to produce distortions in the strain. The exception is model $\mathrm{C}$ in the blue solid line, which has fewer cases than model B in the same curve, but this is purely a fluctuation due to small number statistics. The power-law models are also more likely to produce distortions than the Gaussian models except when comparing models D (Gaussian with 3.3\% dark matter) and $\mathrm{E}$ (power law with $3.3 \%$ dark matter and $\gamma=0$ ), which predict almost identical results, although with the Gaussian model predicting a few more cases with this magnitude in the distortion. Note how models $G$ and $H$ are close to the saturation regime and produce virtually identical results. The plot also shows the gain in probability of distortion when one considers events magnified by larger factors (red curves). If one considers, for instance, the Gaussian model with $1 \%$ dark matter at $\mu=150$ (red curves, model C), between $\approx 20 \%$ and $\approx 30 \%$ of the cases show distortions in the magnification of at least a factor of 4 below $200 \mathrm{~Hz}$. At even larger magnification factors, PBH models with smaller fractions of dark matter would show similar results. Note how the Gaussian model with $0.3 \%$ dark matter as PBHs (model B) is clearly more sensitive than model A (only stars and remnants) despite the fact that model A has a larger surface mass density than model B. However, most of the mass in model A is contained in lowmass stars, for which diffraction effects are small below $200 \mathrm{~Hz}$. It is important to stress that the mass function of the PBHs assumed in this work is not the same as the one assumed for the two components producing the GWs (see Sec. III). In other words, we assume that the merger rate of $\mathrm{BBHs}$ is fixed and independent of the PBHs. For the GWs, we assumed a chirp mass that results from drawing $m_{1}$ and $m_{2}$ from a power law which in our notation would have an exponent $\gamma_{p}=-1.3$. If the GWs considered in this work are sourced by the same PBHs (as already considered by some authors), such a steep mass function would produce, on average, diffraction effects which are smaller than the ones presented in this work for $\gamma_{p}=0,1$, since most of the microlens masses would correspond to smaller objects. On the other hand, smaller masses for the BBHs would result in larger maximum frequencies, where the interference effects are greatest.

Although all the information of the distortion due to microlensing is encapsulated in the relative magnification as a function of the frequency shown in the previous section, it is interesting to show the effect over the observed strain. In Fig. 15, we show examples for the case with magnification $\mu=150$, negative parity, and for the powerlaw mass function model with $\gamma=1$. The plot shows the original signal of an undisturbed strain derived in the postNewtonian approximation as a black solid line. For this strain, we have assumed that $m_{1}=m_{2}=8 \mathrm{M}_{\odot}$ and that the $\mathrm{GW}$ originated at $z=1.5$. The dotted lines show 50 examples of $50 \mathrm{GWs}$ placed at random positions in the source plane. The first three of these 50 examples are 


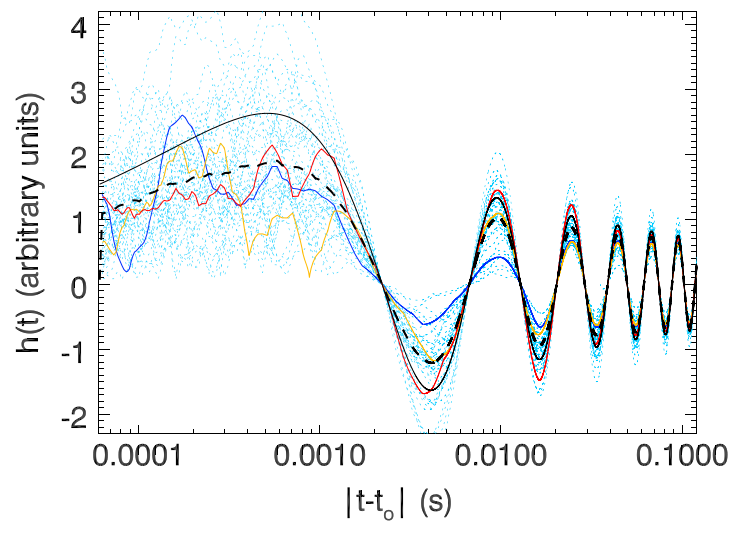

FIG. 15. Distortion of the strain for a lensed GW with $m_{1}=$ $m_{2}=8 \mathrm{M}_{\odot}$ at $z=1.5$ (black solid line). The colored dotted and solid curves correspond to the same cases shown in Fig. 9, that is, the distorted strains due to interference. In all cases, the distortions are more evident toward $\left|t-t_{o}\right|=0$, where $t_{o}$ is the time of merger. The dashed black line shows the average over all random positions, where a systematic damping (compared with the black solid line) of the strain is clearly observed as the frequency of the GW increases (i.e., toward $\left|t-t_{o}\right|=0$ ).

shown as colored solid lines (blue, orange, and red). These 50 curves (and the colored lines) are the same cases shown in Fig. 9 in Sec. VIII A. The time of coalescence takes place at $t=t_{o}$. Note how, in some cases, the amplitude in the last cycles is affected substantially or even suppressed almost completely. Also, it is interesting to see how on average (black dashed line) the amplitude in the last cycles is reduced by some factor that increases with the frequency. This trend was already observed in Figs. 12 and 13, but it is observed more clearly in this plot. The suppression of the last cycles is more prominent in the macroimages with negative parity than in macroimages with positive parity (see [35], where it is also shown how the mean of the magnification remains unchanged when microlenses are present). This follows the expected behavior of lensing in the wave optics regime, where at low frequencies one is expected to be insensitive to the presence of microlenses (i.e., one recovers the mean of the magnification), while, at high frequencies, the GWs can see the microlenses (i.e., the typical magnification tends toward the median of the magnification). If microlensing is predominant in strongly lensed GWs, and as shown by Fig. 15, the last cycle before the ringdown is more likely to be damped (especially in macroimages with negative parity), it is possible that the estimated parameters of the GW (especially the one with negative parity) are biased if microlensing effects are ignored.

We should note that Fig. 15 corresponds to the interference produced in the signal that arrives first and by all multiple images. Since microlensing produces multiple microimages, if the time delay between the first and last microimage is larger than, but comparable to, the inverse of $f_{\text {max }}$, then multiple mergers and ringdowns are expected to be observed. In the simplest case where only two microimages are produced, the $\mathrm{GW}$ from the microimage that arrives first would show interference, but, once the ringdown phase of this GW is over, one may still observe the merger and ringdown of the GW from the second microimage, only this time without any interference.

If one assumes that the BDS model is correct (i.e., all the low-frequency events detected by LIGO are strongly lensed with magnification factors up to several hundreds), this would imply that the fraction of dark matter in the form of PBHs between $5 \mathrm{M}_{\odot}$ and $50 \mathrm{M}_{\odot}$ must be below the $10 \%$ level. Otherwise, about half of the observed lensed GWs would show clear signs of microlensing in the strains. Similarly, if the fraction of dark matter in the form of PBHs in the same mass regime is of the order of, or above, $10 \%$, this would imply that the BDS model cannot be correct and that the observed low-frequency (or high chirp mass) GWs are not strongly lensed. More data and with higher sensitivity (especially at high frequencies) are needed to settle this question.

Future detectors of GWs, such as the Einstein Telescope, will soon increase the sensitivity and extend the range in frequencies where events can be observed. Extending the range toward lower frequencies is interesting, not only for early warning reasons (events can be detected hundreds of seconds before the merger takes place [77]) but also because, at these low frequencies, microlensing effects are expected to be very small, and the parameters estimated at low frequencies can be later used to unveil signs of microlensing at high frequencies. Increasing the sensitivity at high frequencies would allow one to measure, with higher significance, the ringdown (very sensitive to microlensing) and also detect events with smaller chirp masses at cosmological distances. BNSs are expected to be more common than BBHs (the inferred volumetric rate of BNSs at $z=0$ is $\approx 10^{3}$ per year and $\mathrm{Gpc}^{3}$ [78]). However, since the chirp mass is smaller, the signal-to-noise ratio is also smaller than for BBHs, and, hence, they can be observed only at smaller distances (where the optical depth of lensing is small). Once the sensitivity of future GW detectors increases, it will allow one to observe BNSs at larger cosmological distances. If magnified by factors of a few tens to a few hundreds, future observations can reveal lensed GWs from a distant BNS at $z>1$. At higher redshifts, this rate could be even higher, offering a chance of observing these events through lensing. Interestingly, these events, when redshifted (assuming $z \sim 1-2$ ), would appear to be falling within the forbidden mass gap at low mass. Also, the relatively smaller chirp mass results in a larger $f_{\max }$, and it is at these higher frequencies where wave effects are more prominent.

\section{ACKNOWLEDGMENTS}

The author thanks Tom Broadhurst, George Smoot, Bradley Kavanagh, Leo Singer, and Juan Garcia-Bellido 
for useful comments and discussions and Patrick Kelly for gently providing the stellar + remnant mass function. J. M. D. acknowledges the support of Project No. PGC2018101814-B-100 (MCIU/AEI/MINECO/FEDER， UE)
Ministerio de Ciencia, investigación y Universidades. Funded by the Agencia Estatal de Investigación, Unidad de Excelencia María de Maeztu, Ref. No. MDM-2017-0765.
[1] B. J. Carr and S. W. Hawking, Black holes in the early Universe, Mon. Not. R. Astron. Soc. 168, 399 (1974).

[2] B. Carr, F. Kühnel, and M. Sandstad, Primordial black holes as dark matter, Phys. Rev. D 94, 083504 (2016).

[3] J. García-Bellido, A. Linde, and D. Wands, Density perturbations and black hole formation in hybrid inflation, Phys. Rev. D 54, 6040 (1996).

[4] J. García-Bellido, Massive primordial black holes as dark matter and their detection with gravitational waves, J. Phys. Conf. Ser. 840, 012032 (2017).

[5] S. Clesse and J. García-Bellido, Massive primordial black holes from hybrid inflation as dark matter and the seeds of galaxies, Phys. Rev. D 92, 023524 (2015).

[6] M. Y. Khlopov, Primordial black holes, Res. Astron. Astrophys. 10, 495 (2010).

[7] B. J. Kavanagh, D. Gaggero, and G. Bertone, Merger rate of a subdominant population of primordial black holes, Phys. Rev. D 98, 023536 (2018).

[8] S. Deguchi and W. D. Watson, Diffraction in gravitational lensing for compact objects of low mass, Astrophys. J. 307, 30 (1986).

[9] T. T. Nakamura and S. Deguchi, Wave optics in gravitational lensing, Prog. Theor. Phys. Suppl. 133, 137 (1999).

[10] Y. Wang, A. Stebbins, and E. L. Turner, Gravitational Lensing of Gravitational Waves from Merging Neutron Star Binaries, Phys. Rev. Lett. 77, 2875 (1996).

[11] T. T. Nakamura, Gravitational Lensing of Gravitational Waves from Inspiraling Binaries by a Point Mass Lens, Phys. Rev. Lett. 80, 1138 (1998).

[12] M. Sereno, A. Sesana, A. Bleuler, P. Jetzer, M. Volonteri, and M.C. Begelman, Strong Lensing of Gravitational Waves as Seen by LISA, Phys. Rev. Lett. 105, 251101 (2010).

[13] Z. Cao, L.-F. Li, and Y. Wang, Gravitational lensing effects on parameter estimation in gravitational wave detection with advanced detectors, Phys. Rev. D 90, 062003 (2014).

[14] K. K. Y. Ng, K. W. K. Wong, T. Broadhurst, and T. G. F. Li, Precise LIGO lensing rate predictions for binary black holes, Phys. Rev. D 97, 023012 (2018).

[15] S. Jung and C.S. Shin, Gravitational-Wave Fringes at LIGO: Detecting Compact Dark Matter by Gravitational Lensing, Phys. Rev. Lett. 122, 041103 (2019).

[16] J. M. Diego, O. A. Hannuksela, P. L. Kelly, T. Broadhurst, K. Kim, T. G. F. Li, and G. F. Smoot, Observational signatures of microlensing in gravitational waves at LIGO/ Virgo frequencies, Astron. Astrophys. 627, A130 (2019).

[17] J. M. Diego, N. Kaiser, T. Broadhurst, P. L. Kelly, S. Rodney, T. Morishita, M. Oguri, T. W. Ross, A. Zitrin,
M. Jauzac, J. Richard, L. Williams, J. Vega-Ferrero, B. Frye, and A. V. Filippenko, Dark matter under the microscope: Constraining compact dark matter with caustic crossing events, Astrophys. J. 857, 25 (2018).

[18] L. Dai, T. Venumadhav, and K. Sigurdson, Effect of lensing magnification on the apparent distribution of black hole mergers, Phys. Rev. D 95, 044011 (2017).

[19] T. Broadhurst, J. M. Diego, and G. Smoot III, Reinterpreting low frequency LIGO/Virgo events as magnified stellar-mass black holes at cosmological distances, arXiv:1802.05273.

[20] K.-H. Lai, O. A. Hannuksela, A. Herrera-Martín, J. M. Diego, T. Broadhurst, and T. G. F. Li, Discovering intermediate-mass black hole lenses through gravitational wave lensing, Phys. Rev. D 98, 083005 (2018).

[21] L. Dai, S.-S. Li, B. Zackay, S. Mao, and Y. Lu, Detecting lensing-induced diffraction in astrophysical gravitational waves, Phys. Rev. D 98, 104029 (2018).

[22] P. Christian, S. Vitale, and A. Loeb, Detecting stellar lensing of gravitational waves with ground-based observatories, Phys. Rev. D 98, 103022 (2018).

[23] M. Oguri, Effect of gravitational lensing on the distribution of gravitational waves from distant binary black hole mergers, Mon. Not. R. Astron. Soc. 480, 3842 (2018).

[24] X. Li, L. Tang, H.-N. Lin, and L.-L. Wang, Testing the variation of the fine structure constant with strongly lensed gravitational waves, Chin. Phys. C 42, 095104 (2018).

[25] R. Takahashi and T. Nakamura, Wave effects in the gravitational lensing of gravitational waves from chirping binaries, Astrophys. J. 595, 1039 (2003).

[26] C. Cutler and É.E. Flanagan, Gravitational waves from merging compact binaries: How accurately can one extract the binary's parameters from the inspiral waveform?, Phys. Rev. D 49, 2658 (1994).

[27] L.S. Finn, Binary inspiral, gravitational radiation, and cosmology, Phys. Rev. D 53, 2878 (1996).

[28] L. E. Kidder, C. M. Will, and A. G. Wiseman, Coalescing binary systems of compact objects to (post) ${ }^{5 / 2}$-Newtonian order. III. Transition from inspiral to plunge, Phys. Rev. D 47, 3281 (1993).

[29] C. Hanna, M. Megevand, E. Ochsner, and C. Palenzuela, A method for estimating time frequency characteristics of compact binary mergers to improve searches for inspiral, merger and ring-down phases separately, Classical Quantum Gravity 26, 015009 (2009).

[30] One should note that the most likely value for $\mu$ is slightly below 1.0 (i.e., a subtle demagnification) which, when ignored, leads to an overprediction of the distance and, hence, a systematic bias in the estimation of $H_{o}$ if not properly accounted for. 
[31] B. P. Abbott et al. (LIGO Scientific Collaboration and Virgo Collaboration), Binary black hole population properties inferred from the first and second observing runs of advanced LIGO and advanced Virgo, Astrophys. J. Lett. 882, L24 (2019).

[32] P. Kroupa, On the variation of the initial mass function, Mon. Not. R. Astron. Soc. 322, 231 (2001).

[33] S. Clesse and J. García-Bellido, The clustering of massive primordial black holes as dark matter: Measuring their mass distribution with advanced LIGO, Phys. Dark Universe 15, 142 (2017).

[34] T. Broadhurst, J. M. Diego, and G. F. Smoot, A distant origin for magnified LIGO/Virgo black holes implied by binary component masses, arXiv:2002.08821.

[35] J. M. Diego, The Universe at extreme magnification, Astron. Astrophys. 625, A84 (2019).

[36] W. A. Watson, I. T. Iliev, J. M. Diego, S. Gottlöber, A. Knebe, E. Martínez-González, and G. Yepes, Statistics of extreme objects in the Juropa Hubble Volume simulation, Mon. Not. R. Astron. Soc. 437, 3776 (2014).

[37] A. Lapi, M. Negrello, J. González-Nuevo, Z.-Y. Cai, G. De Zotti, and L. Danese, Effective models for statistical studies of galaxy-scale gravitational lensing, Astrophys. J. 755, 46 (2012).

[38] C. Porciani and P. Madau, Gravitational lensing of distant supernovae in cold dark matter universes, Astrophys. J. 532, 679 (2000).

[39] F. Perrotta, C. Baccigalupi, M. Bartelmann, G. De Zotti, and G. L. Granato, Gravitational lensing of extended highredshift sources by dark matter haloes, Mon. Not. R. Astron. Soc. 329, 445 (2002).

[40] S. Hilbert, S. D. M. White, J. Hartlap, and P. Schneider, Strong-lensing optical depths in a $\Lambda \mathrm{CDM}$ universe-II. The influence of the stellar mass in galaxies, Mon. Not. R. Astron. Soc. 386, 1845 (2008).

[41] R. Takahashi, M. Oguri, M. Sato, and T. Hamana, Probability distribution functions of cosmological lensing: Convergence, shear, and magnification, Astrophys. J. 742, 15 (2011).

[42] P. Madau and M. Dickinson, Cosmic star-formation history, Annu. Rev. Astron. Astrophys. 52, 415 (2014).

[43] S. F. Portegies Zwart and S. L. W. McMillan, Black hole mergers in the Universe, Astrophys. J. Lett. 528, L17 (2000).

[44] S. Banerjee, H. Baumgardt, and P. Kroupa, Stellar-mass black holes in star clusters: Implications for gravitational wave radiation, Mon. Not. R. Astron. Soc. 402, 371 (2010).

[45] C. L. Rodriguez, M. Morscher, B. Pattabiraman, S. Chatterjee, C.-J. Haster, and F. A. Rasio, Binary Black Hole Mergers from Globular Clusters: Implications for Advanced LIGO, Phys. Rev. Lett. 115, 051101 (2015).

[46] B. M. Ziosi, M. Mapelli, M. Branchesi, and G. Tormen, Dynamics of stellar black holes in young star clusters with different metallicities-II. Black hole-black hole binaries, Mon. Not. R. Astron. Soc. 441, 3703 (2014).

[47] K. A. Postnov and A. G. Kuranov, Black hole spins in coalescing binary black holes, Mon. Not. R. Astron. Soc. 483, 3288 (2019).

[48] S. Clesse and J. García-Bellido, Detecting the gravitational wave background from primordial black hole dark matter, Phys. Dark Universe 18, 105 (2017).
[49] B. P. Abbott et al. (LIGO Scientific Collaboration and Virgo Collaboration), Search for the isotropic stochastic background using data from Advanced LIGO's second observing run, Phys. Rev. D 100, 061101 (2019).

[50] K. Chang and S. Refsdal, Flux variations of QSO $0957+$ 561 A, B and image splitting by stars near the light path, Nature (London) 282, 561 (1979).

[51] K. Chang and S. Refsdal, Star disturbances in gravitational lens galaxies, Astron. Astrophys. 132, 168 (1984), https://ui .adsabs.harvard.edu/abs/1984A\%26A...132..168C/abstract.

[52] R. Kayser, S. Refsdal, and R. Stabell, Astrophysical applications of gravitational micro-lensing, Astron. Astrophys. 166, 36 (1986), https://ui.adsabs.harvard.edu/abs/ 1986A\%26A...166...36K/abstract.

[53] B. Paczynski, Gravitational microlensing at large optical depth, Astrophys. J. 301, 503 (1986).

[54] P. L. Kelly et al., Extreme magnification of an individual star at redshift 1.5 by a galaxy-cluster lens, Nat. Astron. 2, 334 (2018).

[55] W. Chen, P. L. Kelly, J. M. Diego, M. Oguri, L. L. R. Williams, A. Zitrin, T. L. Treu, N. Smith, T. J. Broadhurst, N. Kaiser, R. J. Foley, A. V. Filippenko, L. Salo, J. Hjorth, and J. Selsing, Searching for highly magnified stars at cosmological distances: Discovery of a redshift 0.94 blue supergiant in archival images of the galaxy cluster MACS J0416.1-2403, Astrophys. J. 881, 8 (2019).

[56] P. Schneider, J. Ehlers, and E. E. Falco, Gravitational Lenses (Springer-Verlag, Berlin, 1992), p. 112.

[57] A. Ulmer and J. Goodman, Femtolensing: Beyond the semiclassical approximation, Astrophys. J. 442, 67 (1995).

[58] O. Hannuksela, K. Haris, K. Ng, S. Kumar, A. Mehta, D. Keitel, T. Li, and P. Ajith, Search for gravitational lensing signatures in ligo-virgo binary black hole events, Astrophys. J. Lett. 874, L2 (2019).

[59] See, however, the discussion above about the reduced probability of observing the counterimage with negative parity.

[60] T. Broadhurst, J. M. Diego, and G. F. Smoot III, Twin LIGO/ Virgo detections of a viable gravitationally-lensed black hole merger, arXiv:1901.03190.

[61] L. P. Singer, D. A. Goldstein, and J. S. Bloom, The two LIGO/Virgo binary black hole mergers on 2019 August 28 were not strongly lensed, arXiv:1910.03601.

[62] S. Klimenko, G. Vedovato, M. Drago, F. Salemi, V. Tiwari, G. A. Prodi, C. Lazzaro, K. Ackley, S. Tiwari, C. F. Da Silva, and G. Mitselmakher, Method for detection and reconstruction of gravitational wave transients with networks of advanced detectors, Phys. Rev. D 93, 042004 (2016).

[63] B. P. Abbott et al. (LIGO Scientific Collaboration and Virgo Collaboration), All-sky search for short gravitational-wave bursts in the second Advanced LIGO and Advanced Virgo run, Phys. Rev. D 100, 024017 (2019).

[64] R. Essick, S. Vitale, E. Katsavounidis, G. Vedovato, and S. Klimenko, Localization of short duration gravitational-wave transients with the early advanced LIGO and Virgo detectors, Astrophys. J. 800, 81 (2015).

[65] S. Hou, X.-L. Fan, K. Liao, and Z.-H. Zhu, Gravitational wave interference via gravitational lensing: Measurements 
of luminosity distance, lens mass, and cosmological parameters, Phys. Rev. D 101, 064011 (2020).

[66] T. Morishita, L. E. Abramson, T. Treu, K. B. Schmidt, B. Vulcani, and X. Wang, Characterizing intracluster light in the hubble frontier fields, Astrophys. J. 846, 139 (2017).

[67] M. Sasaki, T. Suyama, T. Tanaka, and S. Yokoyama, Primordial Black Hole Scenario for the Gravitational-Wave Event GW150914, Phys. Rev. Lett. 117, 061101 (2016).

[68] Y. Ali-Haïmoud, E. D. Kovetz, and M. Kamionkowski, Merger rate of primordial black-hole binaries, Phys. Rev. D 96, 123523 (2017).

[69] L. Liu, Z.-K. Guo, and R.-G. Cai, Effects of the surrounding primordial black holes on the merger rate of primordial black hole binaries, Phys. Rev. D 99, 063523 (2019).

[70] B. V. Lehmann, S. Profumo, and J. Yant, The maximaldensity mass function for primordial black hole dark matter, J. Cosmol. Astropart. Phys. 04 (2018) 007.

[71] H. Niikura, M. Takada, N. Yasuda, R. H. Lupton, T. Sumi, S. More, T. Kurita, S. Sugiyama, A. More, M. Oguri, and M. Chiba, Microlensing constraints on primordial black holes with Subaru/HSC Andromeda observations, Nat. Astron. 3, 524 (2019).

[72] N. Smyth, S. Profumo, S. English, T. Jeltema, K. McKinnon, and P. Guhathakurta, Updated constraints on asteroid-mass primordial black holes as dark matter, Phys. Rev. D 101, 063005 (2020).
[73] B. Carr, M. Raidal, T. Tenkanen, V. Vaskonen, and H. Veermäe, Primordial black hole constraints for extended mass functions, Phys. Rev. D 96, 023514 (2017).

[74] B. Carr, S. Clesse, J. Garcia-Bellido, and F. Kuhnel, Cosmic conundra explained by thermal history and primordial black holes, arXiv:1906.08217.

[75] M. Raidal, C. Spethmann, V. Vaskonen, and H. Veermäe, Formation and evolution of primordial black hole binaries in the early universe, J. Cosmol. Astropart. Phys. 02 (2019) 018.

[76] C. L. Fryer, K. Belczynski, G. Wiktorowicz, M. Dominik, V. Kalogera, and D. E. Holz, Compact remnant mass function: Dependence on the explosion mechanism and metallicity, Astrophys. J. 749, 91 (2012).

[77] K. Cannon, R. Cariou, A. Chapman, M. Crispin-Ortuzar, N. Fotopoulos, M. Frei, C. Hanna, E. Kara, D. Keppel, L. Liao, S. Privitera, A. Searle, L. Singer, and A. Weinstein, Toward early-warning detection of gravitational waves from compact binary coalescence, Astrophys. J. 748, 136 (2012).

[78] B. P. Abbott et al. (LIGO Scientific Collaboration and Virgo Collaboration), GWTC-1: A Gravitational-Wave Transient Catalog of Compact Binary Mergers Observed by LIGO and Virgo during the First and Second Observing Runs, Phys. Rev. X 9, 031040 (2019). 